\title{
Deduction Systems for Coalgebras Over Measurable Spaces
}

\author{
Robert Goldblatt \\ Centre for Logic, Language and Computation \\ Victoria University of Wellington, New Zealand \\ Rob.Goldblatt@vuw.ac.nz
}

21 September, 2008

\begin{abstract}
A theory of infinitary deduction systems is developed for the modal logic of coalgebras for measurable polynomial functors on the category of measurable spaces. These functors have been shown by Moss and Viglizzo to have final coalgebras that represent certain universal type spaces in game-theoretic economics. A notable feature of the deductive machinery is an infinitary Countable Additivity Rule.

A deductive construction of canonical spaces and coalgebras leads to completeness results. These give a proof-theoretic characterisation of the semantic consequence relation for the logic of any measurable polynomial functor as the least deduction system satisfying Lindenbaum's Lemma. It is also the only Lindenbaum system that is sound.

The theory is additionally worked out for Kripke polynomial functors, on the category of sets, that have infinite constant sets in their formation.
\end{abstract}

\section{Introduction}

The theory of coalgebras of certain functors on the category Set of sets [Rut00] provides a coherent framework for the study of structures and state-transition systems of interest to the theory of computation. Relevant functors include those that are polynomial: constructible from constant functors and the identity functor $I d$ by forming products $T_{1} \times T_{2}$, coproducts $T_{1}+T_{2}$, and exponential functors $T^{E}$ with fixed exponent $E$. Coalgebras for polynomial functors can be thought of as generalised deterministic automata, with fixed exponents providing "input" sets, constant functors providing sets of "outputs" or "labels", and the identity functor being associated with a set of states.

A Kripke polynomial functor (KPF) is one constructible by the polynomial operations and the powerset functor $\mathcal{P}$. Coalgebras for KPF's combine the notion of a non-deterministic automaton with that of a Kripke frame for modal logic, resulting in many ideas and techniques from modal logic being adapted to the coalgebraic setting.

A new dimension to this area has now been added by the work of Moss and Viglizzo [MV04, Vig05, MV06], replacing Set by the category Meas of measurable spaces. They study measurable polynomial functors, analogues of KPF's in which $\mathcal{P}$ is replaced by a functor $\Delta$ assigning to each measurable space $\mathbb{X}$ a space $\Delta \mathbb{X}$ whose points are the probability measures on $\mathbb{X}$. They show that the category of coalgebras for any measurable polynomial functor has a final (terminal) object. This is defined by adapting the modal canonical model construction to build 
spaces in which each point is a "truth set", or "description set", the set of all formulas satisfied by some point of some coalgebra. The motivation for this work came from game-theoretic economics, where there are certain "universal type spaces", representing the interactive beliefs of agents, that can be realised as final coalgebras (see [MV04, HS98] for references to the literature on this).

The main purpose of the present paper is to develop a theory of deduction for measurable polynomial functors, investigating the notion $\vdash \varphi$ of a formula $\varphi$ being derivable as a theorem, and more generally the concept of a deducibility relation $\Gamma \vdash \varphi$, capturing the idea that $\varphi$ is deducible from members of a set $\Gamma$ of formulas by means of some axioms and rules of inference. Deduction systems for the logic of a Kripke polynomial functor $T$ were developed by Rößiger [Röß00] and Jacobs [Jac01], providing canonical models and completeness theorems when all constant sets involved in $T$ are finite. In this case, deducibility is finitary in the sense that if $\Gamma \vdash \varphi$, then $\Gamma_{0} \vdash \varphi$ for some finite $\Gamma_{0} \subseteq \Gamma$. The approach taken was many-sorted, with the sorts being the ingredients of $T$, these being the functors involved in the formation of $T$. Each ingredient $S$ has its own set Form $S$ of formulas of sort $S$, and its own deducibility relation $\vdash_{S}$. The many-sorted syntax and semantics was adapted to measurable polynomial functors by Moss and Viglizzo, whose constructions were carried out model-theoretically without the use of proof theory.

When $T$ has an infinite constant ingredient, the modal logic of $T$-coalgebras has a noncompact satisfaction relation, and deducibility is inevitably infinitary. Here we will use a general methodology for infinitary propositional modal proof theory developed in [Gol82] and more extensively in [Gol93]. This was adapted in [FG06] to the logic of polynomial functors, for which a one-sorted language (essentially the formulas of sort $I d$ ) suffices to construct canonical coalgebras that are final. Our aim now is to extend this to the many-sorted context.

There are a number of sources of non-compactness in the coalgebraic logic of a measurable polynomial functor $T$. If a constant space $\mathbb{X}$ is an ingredient of $T$, then all singleton subsets of $\mathbb{X}$ are formulas of sort $\mathbb{X}$. If $\mathbb{X}$ is infinite, then the set of formulas $\Gamma_{\mathbb{X}}=\{\neg\{c\} \mid c \in \mathbb{X}\}$ will be finitely satisfiable, but not satisfiable. Hence $\Gamma_{\mathbb{X}}$ will semantically entail a constant false formula $\perp$, but no finite subset of $\Gamma_{\mathbb{X}}$ will. In our proof theory we will need the deduction relation

$$
\{\neg\{c\} \mid c \in \mathbb{X}\} \vdash_{\mathbb{X}} \perp .
$$

Another source concerns the probability modalities $[\geqslant p]$ that are used to make formulas of sort $\Delta \mathbb{X}$ out of formulas of sort $\mathbb{X}$. A formula $[\geqslant p] \varphi$ can be read "the probability is at least $p$ that $\varphi$ ". If $\varphi$ defines a subset $\llbracket \varphi \rrbracket$ of $\mathbb{X}$, then $[\geqslant p] \varphi$ defines the subset of $\Delta \mathbb{X}$ comprising those measures $\mu$ for which $\mu(\llbracket \varphi \rrbracket) \geqslant p$. There is a well-known observation that $[\geqslant p] \varphi$ follows from all the formulas $[\geqslant q] \varphi$ with $q<p$, but may not follow from any finite number of them. Here we will need the relation

$$
\{[\geqslant q] \varphi \mid q<p\} \vdash_{\Delta \mathbb{X}}[\geqslant p] \varphi
$$

where $p, q$ denote arbitrary rationals in the unit interval $[0,1]$. Note that this observation, and soundness of this rule, depend on the Archimedean property that no real number can be less than $p$ but closer to $p$ than any rational $q<p$.

A third source of non-compactness is the countable additivity of measures. Suppose we have a sequence $\left\{\varphi_{n} \mid n<\omega\right\}$ of formulas that define a non-increasing sequence $\llbracket \varphi_{0} \rrbracket \supseteq \llbracket \varphi_{1} \rrbracket \supseteq \cdots$ of subsets of $\mathbb{X}$ with empty intersection. Then it is a standard fact that the countable additivity of a measure $\mu \in \Delta \mathbb{X}$ implies $\lim _{n \rightarrow \infty} \mu\left(\llbracket \varphi_{n} \rrbracket\right)=0$ (see Lemma 2.1 below). In this situation, for $p>0$ the set $\left\{[\geqslant p] \varphi_{n} \mid n<\omega\right\}$ is not simultaneously satisfiable, while each of its finite subsets may well be. This fact about measures ensures soundness of the rule

$$
\Gamma \vdash_{\mathbb{X}} \perp \text { implies } \quad\left\{[\geqslant p] \varphi \mid \varphi \in \bigwedge_{\omega} \Gamma\right\} \vdash_{\Delta \mathbb{X}} \perp,
$$


where $p>0, \Gamma$ is countable and $\bigwedge_{\omega} \Gamma$ is the set of all conjunctions of finite subsets of $\Gamma$. More strongly, it allows us to formulate a Countable Additivity Rule:

$$
\Gamma \vdash_{\mathbb{X}} \psi \quad \text { implies } \quad\left\{[\geqslant p] \varphi \mid \varphi \in \bigwedge_{\omega} \Gamma\right\} \vdash_{\Delta \mathbb{X}}[\geqslant p] \psi,
$$

for countable $\Gamma$. This rule appears to be new in the literature on finite-formula languages with probabilistic modalities.

Our approach to proof theory is abstract, or "postulational", declaring certain formulas to be axioms and laying down conditions that we require of the relations $\vdash_{S}$, including the Lindenbaum property that every deductively consistent set of formulas has a maximally consistent extension. These maximal sets are then used to proof-theoretically build canonical spaces and a canonical $T$-coalgebra, with the Countable Additivity Rule being needed to prove countable additivity of measures associated with maximal sets of sort $\Delta \mathbb{X}$. From this we obtain a completeness theorem and a verification that these canonical objects are the same as those constructed model-theoretically by Moss and Viglizzo. From the completeness theorem we obtain a purely proof-theoretic characterisation of the semantic consequence relation for the logic of a measurable polynomial functor, as the least deduction system that has the Lindenbaum property (see Theorem 5.17). It is also the only Lindenbaum system that is sound (Corollary 5.15).

The adoption of "Lindenbaum's Lemma" as a postulate rather than a property to be proved is novel and may be a conceptual stumbling block at first. But it is a basic modus operandi of mathematical research to turn a would-be theorem into an axiom. In this case the situation is unavoidable: in [FG06] we showed that a natural deducibility relation for the exponential functor $\omega^{\mathbb{R}}$ on Set fails to be Lindenbaum. This example adapts to Meas, to show that the least deduction system for $\omega^{\mathbb{R}}$ is not Lindenbaum: it has a deductively consistent set of formulas for which the existence of a maximally consistent extension would imply the impossibility that there is an injective function $\mathbb{R} \rightarrow \omega$ (see the end of Section 5).

Sections 2-6 work out this theory for measurable spaces, including showing how an uncountable but separable space like the unit interval $[0,1]$ can be handled by a countable syntax. Sections 7 and 8 adapt the theory to Kripke polynomial functors over Set, and construct a particular system of relations $\vdash_{S}^{+}$that is sound, and can be proven to have the Lindenbaum property when the language is countable. The essential idea is that $\Gamma \vdash_{S}^{+} \varphi$ when $\varphi$ belongs to every "theory" extending $\Gamma$, where a theory is a set of formulas that contains all axioms and is closed under specified inference rules. In the measurable case it is not clear whether this approach produces a system satisfying the Countable Additivity Rule, and this question remains to be resolved.

The paper concludes with brief discussion of some related points, including obstacles to adapting the theory to functors that involve the finitary powerset functor, or discrete probability measures.

\section{Measure-Theoretic Background}

\subsection{The Category of Measurable Spaces}

Let $\mathcal{A}$ be a (Boolean) algebra on a set $X$, i.e. a non-empty collection of subsets of $X$ closed under complements and binary unions. $\mathcal{A}$ is a $\sigma$-algebra if it is also closed under countable unions. Then $\mathbb{X}=(X, \mathcal{A})$ is called a measurable space and the members of $\mathcal{A}$ are its measurable sets. The $\sigma$-algebra of $\mathbb{X}$ will often be denoted $\mathcal{A}_{\mathbb{X}}$.

We write $\sigma\left(\mathcal{A}^{g}\right)$ for the smallest $\sigma$-algebra containing a given set $\mathcal{A}^{g}$ of subsets of $X . \sigma\left(\mathcal{A}^{g}\right)$ is generated by $\mathcal{A}^{g}$, and has the members of $\mathcal{A}^{g}$ as generators. $\mathbb{X}$ is a countably generated measurable space if $\mathcal{A}_{\mathbb{X}}$ has a countable generating set. Later we will present spaces in the form $\mathbb{X}=\left(X, \mathcal{A}_{\mathbb{X}}, \mathcal{A}_{\mathbb{X}}^{g}\right)$ with a designated $\mathcal{A}_{\mathbb{X}}^{g}$ generating $\mathcal{A}_{\mathbb{X}}$. 
A measurable function $f:(X, \mathcal{A}) \rightarrow\left(X^{\prime}, \mathcal{A}^{\prime}\right)$ is a function $f: X \rightarrow X^{\prime}$ that pulls measurable sets back to measurable sets. For this it suffices that $f^{-1}(A) \in \mathcal{A}$ for all sets $A$ in some generating subset of $\mathcal{A}^{\prime}$. The category Meas has the measurable spaces as objects and the measurable functions as morphisms, with the usual functional composition of morphisms.

Any topological space becomes a measurable space by taking its measurable sets to be the Borel sets, the members of the $\sigma$-algebra generated by the open sets (or by the closed sets). So notable objects in Meas include the real line $\mathbb{R}$, the unit interval $[0,1], \mathbb{R}^{n},[0,1]^{n}$, the Hilbert cube $[0,1]^{\omega}$, the Cantor space, and the Baire space $\omega^{\omega}$ - relative to the Borel sets for their usual topologies. As topological spaces these are all Polish: separable and completely metrizable. But it is a theorem of Kuratowski that between any two uncountable Polish spaces there is a Borel isomorphism, a measurable bijection with measurable inverse (see [Sri98, Section 3.3]). Thus from the point of view of the category Meas, these classical spaces are all essentially the same object.

A function $\mu: \mathcal{A} \rightarrow[0, \infty]=\{x \in \mathbb{R} \mid x \geqslant 0\} \cup\{\infty\}$ on an algebra $\mathcal{A}$ is finitely-additive if $\mu\left(A_{1} \cup A_{2}\right)=\mu\left(A_{1}\right)+\mu\left(A_{2}\right)$ whenever $A_{1}$ and $A_{2}$ are disjoint members of $\mathcal{A}$. $\mu$ is countably additive if $\mu\left(\bigcup_{n} A_{n}\right)=\sum_{0}^{\infty} \mu\left(A_{n}\right)$ whenever the sets $\left\{A_{n} \mid n<\omega\right\}$ are pairwise disjoint members of $\mathcal{A}$ whose union $\bigcup_{n} A_{n}$ belongs to $\mathcal{A}$. A measure is a countably additive function with $\mu(\emptyset)=0$. We will need the following basic facts of measure theory, in which a sequence $\left\{A_{n} \mid n<\omega\right\}$ is called non-increasing if $A_{n} \supseteq A_{n+1}$ for all $n$.

Lemma 2.1 Let $\mathcal{A}$ be an algebra.

(1) [Hal74, Theorem 9E] Any measure $\mu$ on $\mathcal{A}$ is continuous from above, meaning that if $\left\{A_{n} \mid n<\omega\right\} \subseteq \mathcal{A}$ is a non-increasing sequence whose intersection belongs to $\mathcal{A}$, with at least one $A_{n}$ having finite measure, then $\mu\left(\bigcap_{n<\omega} A_{n}\right)=\lim _{n \rightarrow \infty} \mu\left(A_{n}\right)$.

(2) [Hal74, Theorem 9F] Let $\mu: \mathcal{A} \rightarrow[0, \infty]$ be finitely additive with $\mu(X)<\infty$. Then $\mu$ is a measure if it is continuous at $\emptyset$, i.e. $\lim _{n \rightarrow \infty} \mu\left(A_{n}\right)=0$ for any non-increasing sequence $\left\{A_{n} \mid n<\omega\right\} \subseteq \mathcal{A}$ with $\bigcap_{n} A_{n}=\emptyset$.

From any measurable space $\mathbb{X}$ we obtain the space $\Delta \mathbb{X}$ of all probability measures on $\mathbb{X}$ : the measures with $\mu(X)=1$. The $\sigma$-algebra of $\Delta \mathbb{X}$ is generated by certain sets $\beta^{p}(A)$ of probability measures, where $p \in[0,1], A \in \mathcal{A}_{\mathbb{X}}$ and $\beta^{p}(A)=\{\mu \mid \mu(A) \geqslant p\}$. But $\beta^{p}(A)=\bigcap\left\{\beta^{q}(A): p>\right.$ $\left.q \in[0,1]_{\mathbb{Q}}\right\}$, where $\mathbb{Q}$ is the rationals and $[0,1]_{\mathbb{Q}}=[0,1] \cap \mathbb{Q}$. Thus the measurable subsets of $\Delta \mathbb{X}$ are generated by the sets $\beta^{q}(A)$ with rational $q$. Moreover, if $\mathcal{A}_{\mathbb{X}}$ is generated by an algebra $\mathcal{A}^{g}$, then the $\sigma$-algebra of $\Delta \mathbb{X}$ is generated by the $\beta^{q}(A)$ 's with $A \in \mathcal{A}^{g}$. This was shown in [HS98] and generalised in [Vig05] to collections $\mathcal{A}^{g}$ that are only closed under finite intersections.

The $\Delta \mathbb{X}$ construction lifts to a functor $\Delta:$ Meas $\rightarrow$ Meas, taking each morphism $f: \mathbb{X} \rightarrow \mathbb{X}^{\prime}$ to $\Delta f: \Delta \mathbb{X} \rightarrow \Delta \mathbb{X}^{\prime}$, defined by $(\Delta f) \mu=\mu \circ f^{-1}$, where $f^{-1}: \mathcal{A}_{\mathbb{X}^{\prime}} \rightarrow \mathcal{A}_{\mathbb{X}}$ maps each measurable set in $\mathbb{X}^{\prime}$ to its inverse image under $f$. The proof that $\Delta f$ is measurable follows from the fact [MV06, Lemma 2.1] that for all $A \in \mathcal{A}_{\mathbb{X}^{\prime}}$,

$$
(\Delta f)^{-1}\left(\beta^{p}(A)\right)=\beta^{p}\left(f^{-1}(A)\right) .
$$

For each functor $T:$ Meas $\rightarrow$ Meas, we write $\Delta T$ for the composite functor $\Delta \circ T:$ Meas $\rightarrow$ Meas.

\subsection{Measurable Functors and Coalgebras}

The Cartesian product $X_{1} \times X_{2}$ of two sets has associated projections $\pi_{j}: X_{1} \times X_{2} \rightarrow X_{j}$ for $j \in\{1,2\}$. The coproduct $X_{1}+X_{2}$ of $X_{1}$ and $X_{2}$ is their disjoint union, with injective insertion functions $i n_{j}: X_{j} \rightarrow X_{1}+X_{2}$ for $j \in\{1,2\}$. Each element of $X_{1}+X_{2}$ is equal to $i n_{j}(x)$ for a 
unique $j$ and a unique $x \in X_{j}$. The $E$-th exponential of a set $X$ is the set $X^{E}$ of all functions from set $E$ to $X$. For each $e \in E$ there is the evaluation-at-e function $e v_{e}: X^{E} \rightarrow X$ having $e v_{e}(f)=f(e)$.

These constructions lift to measurable spaces. The $\sigma$-algebra of the product space $\mathbb{X}_{1} \times \mathbb{X}_{2}$ is generated by the products $A_{1} \times A_{2}$ of measurable sets $A_{j}$ from each factor $\mathbb{X}_{j}$, or equivalently by the inverse images $\pi_{j}^{-1}\left(A_{j}\right)$ of the measurable sets from each factor. The $\sigma$-algebra of the coproduct space $\mathbb{X}_{1}+\mathbb{X}_{2}$ is generated by the insertions $i n_{j}\left(A_{j}\right)$ of the measurable sets $A_{j}$ from each summand $\mathbb{X}_{j}$. The $\sigma$-algebra of the exponential space $\mathbb{X}^{E}$, where the exponent $E$ is any set, is generated by the inverse images $e v_{e}^{-1}(A)$ of the measurable sets $A$ of $\mathbb{X}$ under all evaluation functions. The associated projections, insertions and evaluations are then all measurable functions.

Two measurable functions $f_{1}: \mathbb{X}_{1} \rightarrow \mathbb{X}_{1}^{\prime}$ and $f_{2}: \mathbb{X}_{2} \rightarrow \mathbb{X}_{2}^{\prime}$ have a measurable product $f_{1} \times f_{2}: \mathbb{X}_{1} \times X_{2} \rightarrow \mathbb{X}_{1}^{\prime} \times \mathbb{X}_{2}^{\prime}$ and a measurable coproduct $f_{1}+f_{2}: \mathbb{X}_{1}+\mathbb{X}_{2} \rightarrow \mathbb{X}_{1}^{\prime}+\mathbb{X}_{2}^{\prime}$, where

$$
\begin{aligned}
\left(f_{1} \times f_{2}\right)\left(x_{1}, x_{2}\right) & =\left(f_{1}\left(x_{1}\right), f_{2}\left(x_{2}\right)\right) \\
\left(f_{1}+f_{2}\right)\left(i n_{j}(x)\right) & =i n_{j}\left(f_{j}(x)\right) .
\end{aligned}
$$

The product $T_{1} \times T_{2}$ of two functors on Meas is the functor that acts on spaces by $\mathbb{X} \mapsto T_{1} \mathbb{X} \times T_{2} \mathbb{X}$, and on morphisms by $f \mapsto T_{1}(f) \times T_{2}(f)$. The coproduct functor $T_{1}+T_{2}$ has $\mathbb{X} \mapsto T_{1} \mathbb{X}+T_{2} \mathbb{X}$ and $f \mapsto T_{1}(f)+T_{2}(f)$.

For any function $f: \mathbb{X}_{1} \rightarrow \mathbb{X}_{2}$, there is a function $f^{E}: \mathbb{X}_{1}^{E} \rightarrow \mathbb{X}_{2}^{E}$ defined by $f^{E}(g)=f \circ g$. If $f$ is measurable, then so is $f^{E}$. This follows from the fact that for any subset $A$ of $\mathbb{X}_{2}$,

$$
\left(f^{E}\right)^{-1}\left(e v_{e}^{-1} A\right)=e v_{e}^{-1}\left(f^{-1} A\right) .
$$

The exponential functor $T^{E}$ has $T^{E}(\mathbb{X})=(T \mathbb{X})^{E}$, and $T^{E}(f)=(T f)^{E}$.

Each measurable space $\mathbb{X}$ determines the constant functor on Meas taking all objects to $\mathbb{X}$ and all morphisms to the identity function on $X$. We denote this constant functor also by $\mathbb{X}$. The identity functor on Meas will be denoted $I d$.

A measurable polynomial functor is any functor on Meas that can be constructed in finitely many steps from constant functors and/or the identity functor $I d$ by forming products $T_{1} \times T_{2}$, coproducts $T_{1}+T_{2}$, exponentials $T^{E}$, and measure-space functors $\Delta T$.

For functor $T$ : Meas $\rightarrow$ Meas, a $T$-coalgebra is a pair $(\mathbb{X}, \alpha)$ comprising a measurable space $\mathbb{X}$ and a measurable function $\alpha: \mathbb{X} \rightarrow T \mathbb{X}$. In the coalgebraic approach to systems and processes, points of $\mathbb{X}$ are thought of as states, and $\alpha$ as transition structure. $\alpha(x)$ is a structured organisation of the "next state after $x$ ".

A $T$-coalgebra morphism $f:(\mathbb{X}, \alpha) \rightarrow\left(\mathbb{X}^{\prime}, \alpha^{\prime}\right)$ is given by a Meas-morphism $f: \mathbb{X} \rightarrow \mathbb{X}^{\prime}$ that preserves the transition structures in the sense that $\alpha^{\prime} \circ f=T f \circ \alpha$. The notion of measurable polynomial functor was introduced and developed in [MV04, Vig05, MV06], where it is shown that the category of $T$-coalgebras and $T$-morphisms for a measurable polynomial $T$ has a terminal object, known as a final $T$-coalgebra. ${ }^{1}$

\subsection{The Multigraph of Ingredients}

The ingredients of a measurable polynomial functor $T$ are all the functors involved in the construction of $T$, along with the identity functor. The set $\operatorname{lng} T$ of ingredients can be defined inductively by putting $\operatorname{lng} T=\{T, I d\}$ if $T=I d$ or $T=\mathbb{X} ; \operatorname{lng} T=\{T\} \cup \operatorname{lng} T_{1} \cup \operatorname{lng} T_{2}$ if $T=T_{1} \times T_{2}$ or $T=T_{1}+T_{2} ;$ and $\operatorname{lng} T=\{T\} \cup \operatorname{lng} S$ if $T=S^{E}$ or $T=\Delta S$.

\footnotetext{
${ }^{1}$ [MV04, Vig05, MV06] do not discuss exponential functors, but the theory readily includes them. $\mathbb{X}^{E}$ is essentially a special case of the direct product $\prod_{e \in E} \mathbb{X}_{e}$ with $\mathbb{X}_{e}=\mathbb{X}$.
} 
$\operatorname{lng} T$ is a finite set that includes at least $I d$. We make it into a multigraph with labelled edges $\stackrel{\kappa}{\rightsquigarrow} T$. Here $\kappa$ is one of the constructor symbols $\mathrm{pr}_{1}, \mathrm{pr}_{2}, \mathrm{in}_{1}, \mathrm{in}_{2}, \mathrm{ev}_{e}$, next, $\geqslant p$, where $e$ is an element of some set $E$ occurring as an exponent in $T$, and $p$ is any rational number from $[0,1]$. The constructors $\geqslant p$ will be called probabilistic. The others are definite. The constructor $\geqslant 1$ will be called almost sure.

The edges $\stackrel{\kappa}{\rightsquigarrow} T$ joining ingredients of $T$ are defined by

- $S_{1} \times S_{2} \stackrel{\mathrm{pr}_{j}}{\rightsquigarrow} S_{j}$ and $S_{1}+S_{2} \stackrel{\mathrm{in}_{j}}{\rightsquigarrow} T S_{j}$, for $j \in\{1,2\}$;

- $S^{E \stackrel{\mathrm{ev}_{e}}{\rightsquigarrow} T} S$ for all $e \in E$;

- $\Delta S \stackrel{\geqslant p}{\gg T} S$ for $p \in[0,1]_{\mathbb{Q}}$;

- $I d \stackrel{\text { next }}{\sim} T$.

Note that the only edge in Ing $T$ that depends on $T$ is $\stackrel{\text { next }}{\rightsquigarrow} T$. Without it, the graph would be a

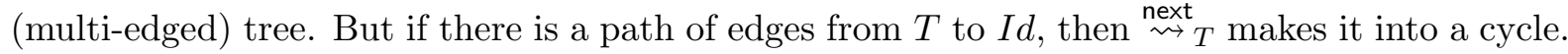

Typically we will drop the $T$-subscripts and write an edge as $\stackrel{\kappa}{\rightsquigarrow}$ when the ambient functor $T$ is understood.

\section{Syntax and Semantics}

Let $T$ be a measurable polynomial functor that remains fixed throughout. Furthermore, suppose that each constant ingredient of $T$ is given by a space presented in the form $\mathbb{X}=\left(X, \mathcal{A}_{\mathbb{X}}, \mathcal{A}_{\mathbb{X}}^{g}\right)$, with $\mathcal{A}_{\mathbb{X}}^{g}$ being a fixed generating set for $\mathcal{A}_{\mathbb{X}}$.

We define a many-sorted modal language for $T$-coalgebras, of the kind developed for certain functors on Set in [Röß00, Jac01] and adapted to Meas in [MV04]. The sorts will be the ingredients of $T$. Each constructor symbol $\kappa$ has a modal connective $[\kappa]$, of "box" type. For each edge $S \stackrel{\kappa}{\rightsquigarrow} S^{\prime}$ in $\operatorname{lng} T,[\kappa]$ makes formulas of sort $S$ out of formulas of sort $S^{\prime}$. The notation $\varphi: S$ means that $\varphi$ is a formula of sort $S$. The set of all formulas of sort $S$ will be denoted Form $_{S}$. The notation $\Gamma: S$ means that $\Gamma$ is a set of formulas of sort $S$, i.e. $\Gamma \subseteq$ Form $_{S}$.

Amongst the formulas of constant sort $\mathbb{X}$ will be the measurable generators from $\mathcal{A}_{\mathbb{X}}^{g}$. The notation $\varphi:: S$ is used to mean that $\varphi: S$ and every subformula of $\varphi$ of constant sort is a measurable set. $\Gamma:: S$ means that $\varphi:: S$ for all $\varphi \in \Gamma$.

Here are the formation rules for formulas, in which $S$ denotes an arbitrary ingredient of $T$.

- $\perp_{S}: S$.

- $A: \mathbb{X}$ if $A \in \mathcal{A}_{\mathbb{X}}^{g}$ or $A$ is a singleton subset of $\mathbb{X}$.

- If $\varphi_{1}: S$ and $\varphi_{2}: S$, then $\varphi_{1} \rightarrow \varphi_{2}: S$.

- If $S \stackrel{\kappa}{\rightsquigarrow} S^{\prime}$ in $\operatorname{lng} T$ with $\kappa \neq(\geqslant p)$, and $\varphi: S^{\prime}$, then $[\kappa] \varphi: S$.

- If $\Delta S \in \operatorname{lng} T$ and $\varphi:: S$, then $[\geqslant p] \varphi: \Delta S$ for any $p \in[0,1]_{\mathbb{Q}}$.

The probability modality $[\geqslant p]$ can be read "the probability is at least $p$ that ...". The inclusion of singletons as formulas of constant sort allows us to "name" the elements of a constant space $\mathbb{X}$. This will be needed later in the construction of canonical coalgebras (see the definition of the measurable maps $r_{\mathbb{X}}$ in the proof of Lemma 5.7).

The Boolean connectives $\neg, \wedge, \vee, \leftrightarrow$ are defined from $\rightarrow$ and $\perp_{S}$ in the usual way; in particular $\neg \varphi$ is $\varphi \rightarrow \perp_{S}$ for $\varphi: S$. $\top_{S}$ is defined to be $\neg \perp_{S}$. We write $\bigwedge \Gamma$ for the conjunction of a finite set $\Gamma$ of formulas, in particular taking $\bigwedge\{\varphi\}=\varphi$ and $\bigwedge \emptyset=\top_{S}$. 
In any $T$-coalgebra $(\mathbb{X}, \alpha)$, each formula $\varphi: S$ is interpreted as a subset $\llbracket \varphi \rrbracket_{S}^{\alpha}$ of $S \mathbb{X}$, thought of as the set of points in the space $S \mathbb{X}$ that satisfy $\varphi$. This is defined inductively as follows, using $X \Rightarrow Y=(-X) \cup Y$ :

$$
\begin{aligned}
\llbracket \perp_{S} \rrbracket_{S}^{\alpha} & =\emptyset \\
\llbracket A \rrbracket_{\mathbb{X}^{\prime}}^{\alpha} & =A \\
\llbracket \varphi_{1} \rightarrow \varphi_{2} \rrbracket_{S}^{\alpha} & =\llbracket \varphi_{1} \rrbracket_{S}^{\alpha} \Rightarrow \llbracket \varphi_{2} \rrbracket_{S}^{\alpha} \\
\llbracket\left[\mathrm{pr}_{j}\right] \varphi \rrbracket_{S_{1} \times S_{2}}^{\alpha} & =\pi_{j}^{-1} \llbracket \varphi \rrbracket_{S_{j}}^{\alpha} \\
\llbracket\left[\mathrm{in}_{1}\right] \varphi \rrbracket_{S_{1}+S_{2}}^{\alpha} & =i n_{1}\left(\llbracket \varphi \rrbracket_{S_{1}}^{\alpha}\right) \cup i n_{2}\left(S_{2} \mathbb{X}\right) \\
\llbracket\left[\mathrm{in}_{2}\right] \varphi \rrbracket_{S_{1}+S_{2}}^{\alpha} & =i n_{1}\left(S_{1} \mathbb{X}\right) \cup i n_{2}\left(\llbracket \varphi \rrbracket_{S_{2}}^{\alpha}\right) \\
\llbracket\left[\mathrm{ev}_{e}\right] \varphi \rrbracket_{S^{E}}^{\alpha} & =e v_{e}^{-1} \llbracket \varphi \rrbracket_{S}^{\alpha} \\
\llbracket[\mathrm{next}] \varphi \rrbracket_{I d}^{\alpha} & =\alpha^{-1} \llbracket \varphi \rrbracket_{T}^{\alpha} \\
\llbracket[\geqslant p] \varphi \rrbracket_{\Delta S}^{\alpha} & =\beta^{p} \llbracket \varphi \rrbracket_{S}^{\alpha} .
\end{aligned}
$$

An inductive argument shows that if $\varphi:: S$, then $\llbracket \varphi \rrbracket_{S}^{\alpha}$ is measurable. This is where the restriction on formation of $[\geqslant p] \varphi$ to $\varphi:: S$ is needed. ${ }^{2}$ We will take it as understood that $\varphi:: S$ whenever the notation $[\geqslant p] \varphi$ is used.

Satisfaction relations reminiscent of Kripkean modal semantics can be introduced by writing $\alpha, x \models_{S} \varphi$ to mean that $x \in \llbracket \varphi \rrbracket_{S}^{\alpha}$. This gives

$$
\begin{array}{lll}
\alpha, x \not \models_{S} \perp_{S} & & \\
\alpha, x \models_{\mathbb{X}^{\prime}} A & \text { iff } \quad x \in A \\
\alpha, x \models_{S} \varphi_{1} \rightarrow \varphi_{2} & \text { iff } \quad \alpha, x \models_{S} \varphi_{1} \text { implies } \alpha, x \models_{S} \varphi_{2} \\
\alpha, x \models_{S_{1} \times S_{2}}\left[\mathrm{pr}_{j}\right] \varphi & \text { iff } \quad \alpha, \pi_{j}(x) \models_{S_{j}} \varphi \\
\alpha, x=_{S_{1}+S_{2}}\left[\mathrm{in}_{j}\right] \varphi & \text { iff } \quad x=i n_{j}(y) \text { implies } \alpha, y \models_{S_{j}} \varphi \\
\alpha, f \models_{S^{E}}\left[\mathrm{ev}_{e}\right] \varphi & \text { iff } \quad \alpha, f(e) \models_{S} \varphi \\
\alpha, x=_{I d}[\mathrm{next}] \varphi & \text { iff } \quad \alpha, \alpha(x) \models_{T} \varphi \\
\alpha, \mu=_{\Delta S}[\geqslant p] \varphi & \text { iff } \quad \mu\left(\llbracket \varphi \rrbracket_{S}^{\alpha}\right) \geqslant p .
\end{array}
$$

Note that the clauses for the definite modalities $[\kappa]$ all have the familiar relational form

$$
\alpha, x \models_{S}[\kappa] \varphi \quad \text { iff } \quad x R_{\kappa} y \text { implies } \alpha, y \models_{S^{\prime}} \varphi
$$

where $R_{\kappa}$ is a binary relation that is functional: each $x$ is $R_{\kappa}$-related to at most one $y$. Here $x R_{\pi_{j}} y$ iff $y=\pi_{j}(x), x R_{\text {next }} y$ iff $y=\alpha(x)$, and these relations are total. On the other hand $x R_{\mathrm{in}_{j}} y$ iff $y=\operatorname{in}_{j}(x)$, and the domain of $R_{\mathrm{in}_{j}}$ is the copy $i n_{j}\left(S_{j} X\right)$ of $S_{j} X$ within $S_{1} X+S_{2} X$.

Satisfaction is defined for formula-sets $\Gamma: S$ by putting $\alpha, x \models_{S} \Gamma$ iff for all $\varphi \in \Gamma, \alpha, x \models_{S} \varphi$. Local and global semantic consequence relations are then defined, for $\Gamma \cup\{\varphi\} \subseteq$ Form $_{S}$, by

$$
\begin{array}{lll}
\Gamma \models_{S}^{\alpha} \varphi & \text { iff } & \text { for all } x \text { in } S \mathbb{X}, \alpha, x \models_{S} \Gamma \text { implies } \alpha, x \models_{S} \varphi ; \\
\Gamma \models_{S} \varphi & \text { iff } \quad \Gamma \models_{S}^{\alpha} \varphi \text { for all } T \text {-coalgebras } \alpha .
\end{array}
$$

\footnotetext{
${ }^{2}$ The syntax of [MV06] is slightly different. In place of $\left[\mathrm{pr}_{j}\right]$ it has formulas $\left\langle\varphi_{1}, \varphi_{2}\right\rangle_{S_{1} \times S_{2}}: S_{1} \times S_{2}$ for $\varphi_{j}: S_{j}$, equivalent to $\left[\mathrm{pr}_{1}\right] \varphi_{1} \wedge\left[\mathrm{pr}_{2}\right] \varphi_{2}$. In place of $\left[\mathrm{in}_{j}\right]$ it uses the formation $\operatorname{inj}_{S_{1}+S_{2}} \varphi_{j}: S_{1}+S_{2}$ for $\varphi_{j}: S_{j}$. This is effectively the "diamond dual" to $\left[\mathrm{in}_{j}\right]$, and is equivalent to $\neg\left[\mathrm{in}_{j}\right] \perp \wedge\left[\mathrm{in}_{j}\right] \varphi_{j}$ in the present semantics.
} 
We say that $\varphi: S$ is valid in the coalgebra $\alpha$, written $\alpha \models_{S} \varphi$, if $\alpha, x \models_{S} \varphi$ for all $x$ in $S \mathbb{X}$. Equivalently, this says both that $\llbracket \varphi \rrbracket_{S}^{\alpha}=S X$ and that $\emptyset \models_{S}^{\alpha} \varphi$. A set $\Gamma: S$ is satisfiable if $\alpha, x \models_{S} \Gamma$ for some $x$ in some $\alpha$. Equivalently this means that $\Gamma \not \nvdash_{S} \perp_{S}$.

Now define

$$
\operatorname{des}_{S}^{\alpha}(x)=\left\{\varphi: S \mid \alpha, x=_{S} \varphi\right\} .
$$

$\operatorname{des}_{S}^{\alpha}(x)$ is the $S$-description set of $x$ in $\alpha$. Such sets are often called "truth sets" in traditional modal semantics. Our aim is to provide a proof-theoretic characterisation of them.

\section{T-Deduction Systems}

We take an approach to infinitary proof theory that was developed for propositional modal logics in [Gol82, Gol93] and adapted to polynomial coalgebraic logic in [FG06]. As explained in the Introduction, the fundamental notion is a "deducibility relation" $\Gamma \vdash \varphi$, from sets of formulas to formulas, that is intended to capture the idea that $\varphi$ is deducible from members of $\Gamma$ with the aid of various axioms and rules of inference. The definition of $\vdash$ is to be syntactic, depending only on the symbolic pattern of formulas and basic set-theoretic properties of sets of them. We then attempt to show that $\vdash$ is identical to some semantically defined consequence relation $\models$, thereby characterising $\mid=$ proof-theoretically.

In our present context we need a many-sorted system $\left\{\vdash_{S} \mid S \in \operatorname{lng} T\right\}$ of relations, with $\vdash_{S}$ being a relation from the powerset $\mathcal{P}\left(\right.$ Form $\left._{S}\right)$ to Form $_{S}$. The notion of a $T$-deduction system is defined by declaring certain formulas to be axioms and laying down conditions that we require of the relations $\vdash_{S}$, including the Lindenbaum property that every deductively consistent set of formulas has a maximally consistent extension. These maximal sets are then used to build a canonical $T$-coalgebra for which they turn out to be the description sets. This leads to a characterisation of the semantic consequence relation system $\left\{\models_{S} \mid S \in \operatorname{lng} T\right\}$ as the least system that satisfies our deducibility postulates and the Lindenbaum property. It is also shown to be the only such system that is sound.

Definition 4.1 (Axioms) The set $A x_{S} \subseteq$ Form $_{S}$ of $S$-axioms is defined, for all $S \in \operatorname{lng} T$, to consist of the following formulas.

1. All Boolean tautologies $\varphi: S$.

2. For $S=\mathbb{X}, A: \mathbb{X}$ and $c \in X$,
(a) $\{c\} \rightarrow A \quad$ if $c \in A$
(b) $\{c\} \rightarrow \neg A \quad$ if $c \notin A$

3. For $S=S_{1} \times S_{2}, j \in\{1,2\}$ and $\varphi: S_{j}$,
(a) $\neg\left[\mathrm{pr}_{j}\right] \varphi \rightarrow\left[\mathrm{pr}_{j}\right] \neg \varphi$
(b) $\neg\left[\operatorname{pr}_{j}\right] \perp_{S_{j}}$

4. For $S=S_{1}+S_{2}$,
(a) $\neg\left[\mathrm{in}_{j}\right] \varphi \rightarrow\left[\mathrm{in}_{j}\right] \neg \varphi$
(b) $\neg\left[\mathrm{in}_{1}\right] \perp_{S_{1}} \leftrightarrow\left[\mathrm{in}_{2}\right] \perp_{S_{2}}$

5. For $S=U^{E}$ and $\varphi: U$,

(a) $\neg\left[\mathrm{ev}_{e}\right] \varphi \rightarrow\left[\mathrm{ev}_{e}\right] \neg \varphi$ 

(b) $\neg\left[\mathrm{ev}_{e}\right] \perp_{U}$

6. For $S=I d$ and $\varphi: T$,

(a) $\neg[$ next $] \varphi \rightarrow[$ next $] \neg \varphi$

(b) $\neg[$ next $] \perp_{T}$

7. For $S=\Delta S^{\prime}$,

(a) $[\geqslant 1](\varphi \rightarrow \psi) \rightarrow([\geqslant p] \varphi \rightarrow[\geqslant p] \psi)$

(b) $[\geqslant p] \top S^{\prime}$

(c) $[\geqslant p] \neg \varphi \rightarrow \neg[\geqslant q] \varphi \quad$ if $p+q>1$

(d) $[\geqslant p](\varphi \wedge \psi) \wedge[\geqslant q](\varphi \wedge \neg \psi) \rightarrow[\geqslant p+q] \varphi \quad$ if $p+q \leqslant 1$

(e) $\neg[\geqslant p] \varphi \wedge \neg[\geqslant q] \psi \rightarrow \neg[\geqslant p+q](\varphi \vee \psi) \quad$ if $p+q \leqslant 1$

These axioms express evident properties of a $T$-coalgebra. In axiom-groups $3-6$ for the definite modalities $[\kappa]$, axiom (a) corresponds to functionality of the relation $R_{\kappa}$ of (3.1). Axioms (3b), (5b) and (6b) correspond to the totality of $R_{\kappa}$, and (4b) expresses the fact that $\left(S_{1}+S_{2}\right) \mathbb{X}$ is the disjoint union of $i n_{1}\left(S_{1} X\right)=\llbracket \neg\left[\mathrm{in}_{1}\right] \perp_{S_{1}} \rrbracket$ and $i n_{2}\left(S_{2} X\right)=\llbracket \neg\left[\mathrm{in}_{2}\right] \perp_{S_{2}} \rrbracket$ in any coalgebra on $\mathbb{X}$. These can also be written as $i_{1}\left(S_{1} X\right)=\llbracket\left[i n_{2}\right] \perp_{S_{2}} \rrbracket$ and $i n_{2}\left(S_{2} X\right)=\llbracket\left[i n_{1}\right] \perp_{S_{1}} \rrbracket$.

The axioms of group 7 expresses properties of finitely additive probability measures. Axioms (7b)-(7d) appear in [HM01] as part of an axiom system for a propositional logic with probabilistic modalities that is attributed to R. J. Aumann. ${ }^{3}$ (7e) is validated by the general inequality $\mu(A)+\mu(B) \geqslant \mu(A \cup B)$. In place of $(7 \mathrm{e})$, Aumann's system has the axiom

$$
\neg[\geqslant p](\varphi \wedge \psi) \wedge \neg[\geqslant q](\varphi \wedge \neg \psi) \rightarrow \neg[\geqslant p+q] \varphi \quad \text { if } p+q \leqslant 1,
$$

which corresponds to the weaker assertion of this inequality for disjoint $A$ and $B$. (7c) and (7d) together correspond to the reverse inequality $\mu(A)+\mu(B) \leqslant \mu(A \cup B)$ for disjoint $A$ and $B$. See Lemma 4.7 and Theorem 5.4 for the use of these axioms in constructing finitely-additive measures (similar axioms for probability quantifiers in predicate languages can be found in [Hoo78], including axiom (7a)).

With these remarks in mind, it is left to the reader to complete the verification of

Theorem 4.2 For any $S \in \operatorname{lng} T$, all $S$-axioms are valid in all $T$-coalgebras.

\section{Definition 4.3 (Notation for formula-sets)}

- $\Sigma \subseteq_{\omega} \Gamma$ means that $\Sigma$ is a finite subset of $\Gamma$.

- $\bigwedge_{\omega} \Gamma$ is the set $\left\{\bigwedge \Sigma \mid \Sigma \subseteq_{\omega} \Gamma\right\}$ of conjunctions of all finite subsets of $\Gamma$.

- $\psi \rightarrow \Gamma=\{\psi \rightarrow \varphi \mid \varphi \in \Gamma\}$.

- For each edge $S \stackrel{\kappa}{\rightsquigarrow} S^{\prime}$ and $\Gamma: S^{\prime}$, define $[\kappa] \Gamma=\{[\kappa] \varphi \mid \varphi \in \Gamma\}: S$.

\section{Definition 4.4 (Deduction systems)}

Let $D=\left\{\vdash_{S} \mid S \in \operatorname{lng} T\right\}$ be a collection of relations $\vdash_{S} \subseteq \mathcal{P}\left(\right.$ Form $\left._{S}\right) \times$ Form $_{S}$. Then $D$ is a $T$-deduction system if the following hold for all ingredients $S$ :

- Assumption Rule: $\varphi \in \Gamma \cup A x_{S}$ implies $\Gamma \vdash_{S} \varphi$.

\footnotetext{
${ }^{3}$ In a 1995 discussion paper whose published version [Aum99] does not contain the axiom system.
} 
- Modus Ponens: $\{\varphi, \varphi \rightarrow \psi\} \vdash_{S} \psi$.

- Cut Rule: If $\Gamma \vdash_{S} \psi$ for all $\psi \in \Sigma$ and $\Sigma \vdash_{S} \varphi$, then $\Gamma \vdash_{S} \varphi$.

- Deduction Rule: $\Gamma \cup\{\varphi\} \vdash_{S} \psi$ implies $\Gamma \vdash_{S} \varphi \rightarrow \psi$.

- Constant Rule: If $\mathbb{X} \in \operatorname{lng} T, \quad\{\neg\{c\} \mid c \in X\} \vdash_{\mathbb{X}} \perp_{\mathbb{X}}$.

- Definite Box Rule: For each edge $S \stackrel{\kappa}{\rightsquigarrow} S^{\prime}$ in $\operatorname{lng} T$ with $\kappa$ a definite constructor, $\Gamma \vdash_{S^{\prime}} \psi$ implies $[\kappa] \Gamma \vdash_{S}[\kappa] \psi$.

- Archimedean Rule: If $\Delta S \in \operatorname{lng} T, \quad\{[\geqslant q] \varphi \mid q<p\} \vdash_{\Delta S}[\geqslant p] \varphi$.

- Countable Additivity Rule: If $\Delta S \in \operatorname{lng} T$, then for countable $\Gamma:: S$, $\Gamma \vdash_{S} \psi$ implies $[\geqslant p]\left(\bigwedge_{\omega} \Gamma\right) \vdash_{\Delta S}[\geqslant p] \psi$.

The following properties of a deduction system can be proven as in [Gol93, Lemmas 9.2.1, 9.2.2].

\section{Lemma 4.5}

(1) Monotonicity: If $\Gamma \vdash_{S} \varphi$ and $\Gamma \subseteq \Delta$, then $\Delta \vdash_{S} \varphi$.

(2) Detachment: If $\Gamma \vdash_{S} \varphi$ and $\Gamma \vdash_{S} \varphi \rightarrow \psi$, then $\Gamma \vdash_{S} \psi$.

(3) Converse Deduction: $\Gamma \vdash_{S} \varphi \rightarrow \psi$ implies $\Gamma \cup\{\varphi\} \vdash_{S} \psi$.

(4) If $\Gamma \vdash_{S} \varphi$ and $\Gamma \cup\{\varphi\} \vdash_{S} \perp$, then $\Gamma \vdash_{S} \perp$.

(5) $\Gamma \cup\{\neg \varphi\} \vdash_{S} \perp_{S}$ iff $\Gamma \vdash_{S} \varphi$.

(6) Implication Rule: $\Gamma \vdash_{S} \varphi$ implies $\psi \rightarrow \Gamma \vdash_{S} \psi \rightarrow \varphi$.

The next Lemma derives some modal principles. Here we write $\vdash_{S} \psi$ to mean that $\emptyset \vdash_{S} \psi$, and $\varphi \vdash_{S} \psi$ to mean that $\{\varphi\} \vdash_{S} \psi$.

\section{Lemma 4.6}

(1) $\vdash_{\Delta S}[\geqslant 0] \varphi$.

(2) $\vdash_{\Delta S} \neg[\geqslant q] \perp_{S} \quad$ if $q>0$.

For each edge $S \stackrel{\kappa}{\rightsquigarrow} S^{\prime}$ in $\operatorname{lng} T$,

$(3) \vdash_{S^{\prime}} \psi$ implies $\vdash_{S}[\kappa] \psi$;

(4) $\vdash_{S}[\kappa](\varphi \rightarrow \psi) \rightarrow([\kappa] \varphi \rightarrow[\kappa] \psi)$, if $\kappa$ is definite or almost sure;

$(5) \vdash_{S^{\prime}} \varphi \rightarrow \psi$ implies $\vdash_{S}[\kappa] \varphi \rightarrow[\kappa] \psi$;

(6) $\vdash_{S^{\prime}} \varphi \leftrightarrow \psi$ implies $\vdash_{S}[\kappa] \varphi \leftrightarrow[\kappa] \psi$.

(7) $\vdash_{\Delta S}[\geqslant q] \varphi \rightarrow[\geqslant p] \varphi \quad$ if $q>p$.

(8) Almost Sure Box Rule: If $\Delta S \in \operatorname{lng} T$ and $\Gamma:: S$ is countable, then $\Gamma \vdash_{S} \psi$ implies $[\geqslant 1] \Gamma \vdash_{\Delta S}[\geqslant 1] \psi$.

Proof. 
(1) By the Archimedean Rule, as $\{[\geqslant 0] \varphi \mid q<0\}=\emptyset$.

(2) If $q>0$, axiom (7c) gives $\vdash_{\Delta S}[\geqslant 1] \neg \perp_{S} \rightarrow \neg[\geqslant q] \perp_{S}$. But $\vdash_{\Delta S}[\geqslant 1] \neg \perp_{S}$ by axiom (7b).

(3) If $\kappa$ is definite, put $\Gamma=\emptyset$ in the Definite Box Rule and note $[\kappa] \emptyset=\emptyset$. If $\kappa$ is probabilistic, and $\vdash_{S^{\prime}} \psi$, putting $\Gamma=\emptyset$ in the Countable Additivity Rule gives $[\kappa] \top_{S^{\prime}} \vdash_{S}[\kappa] \psi$ (since $\left.\top_{S^{\prime}}=\bigwedge \emptyset\right)$. But $\vdash_{S}[\kappa] \top_{S^{\prime}}$ from axiom (7b), so the Deduction and Detachment Rules then give $\vdash_{S}[\kappa] \psi$.

(4) If $\kappa$ is definite, apply the Definite Box Rule to Modus Ponens to obtain $\{[\kappa](\varphi \rightarrow \psi),[\kappa] \varphi\} \vdash_{S}$ $[\kappa] \psi$. Then two applications of the Deduction Rule give the desired result.

If $\kappa$ is almost sure, the result comes from axiom (7a) with $p=1$.

(5) Let $\vdash_{S^{\prime}} \varphi \rightarrow \psi$. Then if $\kappa$ is definite, $\vdash_{S}[\kappa](\varphi \rightarrow \psi)$ by (3), and so the result follows from (4) by Detachment. But if $\kappa$ is probabilistic, with $S=\Delta S^{\prime}$, then $\vdash_{S}[\geqslant 1](\varphi \rightarrow \psi)$ by $(3)$, and $\vdash_{S}[\geqslant 1](\varphi \rightarrow \psi) \rightarrow([\kappa] \varphi \rightarrow[\kappa] \psi)$ by axiom $(7 \mathrm{a})$, so again the result follows.

(6) By (5) and Boolean reasoning.

(7) If $q>p$, then by axiom (7e),

$$
\vdash_{\Delta S} \neg[\geqslant p] \varphi \wedge \neg[\geqslant q-p] \perp_{S} \rightarrow \neg[\geqslant q]\left(\varphi \vee \perp_{S}\right) .
$$

Using (6) and Boolean reasoning, this gives

$$
\vdash_{\Delta S} \neg[\geqslant q-p] \perp_{S} \rightarrow(\neg[\geqslant p] \varphi \rightarrow \neg[\geqslant q] \varphi) .
$$

Since $\vdash_{\Delta S}[\geqslant q-p] \perp_{S}$ by (2), further Boolean reasoning gives the desired $\vdash_{\Delta S}[\geqslant q] \varphi \rightarrow$ $[\geqslant p] \varphi$.

(8) From $\Gamma \vdash_{S} \psi$, the Countable Additivity Rule gives $[\geqslant 1]\left(\bigwedge_{\omega} \Gamma\right) \vdash_{\Delta S}[\geqslant 1] \psi$. But by part (3) with $\kappa=(\geqslant 1)$ and axiom (7a) with $p=1$, standard modal reasoning gives $\vdash_{\Delta S} \bigwedge[\geqslant 1] \Gamma_{0} \rightarrow$ $[\geqslant 1] \wedge \Gamma_{0}$ for all $\Gamma_{0} \subseteq_{\omega} \Gamma$. Hence $[\geqslant 1] \Gamma \vdash_{\Delta S} \varphi$ for all $\varphi \in[\geqslant 1]\left(\bigwedge_{\omega} \Gamma\right)$. The Cut Rule then gives $[\geqslant 1] \Gamma \vdash_{\Delta S}[\geqslant 1] \psi$.

Lemma 4.7 Suppose $\Delta S \in \operatorname{lng} T$ and $\vdash_{S} \neg(\varphi \wedge \psi)$. Then

(1) $\vdash_{\Delta S}[\geqslant p] \varphi \rightarrow \neg[\geqslant q] \psi \quad$ if $p+q>1$.

$(2) \vdash_{\Delta S}[\geqslant p] \varphi \wedge[\geqslant q] \psi \rightarrow[\geqslant p+q](\varphi \vee \psi) \quad$ if $p+q \leqslant 1$.

Proof.

(1) $\vdash_{\Delta S}[\geqslant p] \varphi \rightarrow[\geqslant p] \neg \neg \varphi$ by Lemma 4.6(5), and $[\geqslant p] \neg \neg \varphi \rightarrow \neg[\geqslant q] \neg \varphi$ by axiom (7c). But if $\vdash_{S} \neg(\varphi \wedge \psi)$ then $\vdash_{S} \psi \rightarrow \neg \varphi$, hence $\vdash_{\Delta S}[\geqslant q] \psi \rightarrow[\geqslant q] \neg \varphi$, so $\vdash_{\Delta S} \neg[\geqslant q] \neg \varphi \rightarrow \neg[\geqslant q] \psi$.

(2) In axiom (7d), simultaneously replace $\varphi$ by $\varphi \vee \psi$, and $\psi$ by $\varphi$. Now $\vdash_{S}((\varphi \vee \psi) \wedge \varphi) \leftrightarrow \varphi$, so by Lemma 4.6(6), $\vdash_{\Delta S}[\geqslant p]((\varphi \vee \psi) \wedge \varphi) \leftrightarrow[\geqslant p] \varphi$. But when $\vdash_{S} \neg(\varphi \wedge \psi), \vdash_{S}((\varphi \vee \psi) \wedge \neg \varphi) \leftrightarrow \psi$, hence $\vdash_{\Delta S}[\geqslant q]((\varphi \vee \psi) \wedge \neg \varphi) \leftrightarrow[\geqslant q] \psi$. Therefore (2) follows from this instance of axiom $(7 \mathrm{~d})$.

\section{Theorem 4.8}


(1) For any $T$-coalgebra $(\mathbb{X}, \alpha)$, the system Conseq $_{T}^{\alpha}=\left\{=_{S}^{\alpha} \mid S \in \operatorname{Ing} T\right\}$ of local consequence relations is a $T$-deduction system.

(2) The global system Conseq $_{T}=\left\{\models_{S} \mid S \in \operatorname{Ing} T\right\}$ is a T-deduction system.

Proof. (2) follows readily from (1). For (1) we have to show that the relations $\models_{S}^{\alpha}$ satisfy the rules of Definition 4.4. Much of this is straightforward (recall Theorem 4.2), and we focus on the rules for the probability modalities.

That $\{[\geqslant q] \varphi \mid q<p\}=_{\Delta S}^{\alpha}[\geqslant p] \varphi$ is immediate from the Archimedean property of $[0,1]$ that no real number can be less than $p$ but closer to $p$ than any rational $q<p$.

We discuss the Countable Additivity Rule in more detail. Let $\Gamma \models_{S}^{\alpha} \varphi$ for a countable $\Gamma:: S$. If $\Gamma=\emptyset$, then $\alpha=_{S} \varphi$, so $\mu\left(\llbracket \varphi \rrbracket_{S}^{\alpha}\right)=1$ for all $\mu \in \Delta S X$, hence $\alpha \models_{\Delta S}[\geqslant p] \varphi$ as desired.

Suppose then that $\Gamma \neq \emptyset$, say $\Gamma=\left\{\varphi_{n} \mid n<\omega\right\}$. Put $\psi_{n}=\varphi_{0} \wedge \cdots \wedge \varphi_{n}$. Then $\left\{\llbracket \psi_{n} \rrbracket_{S}^{\alpha} \mid n<\omega\right\}$ is a non-increasing sequence of measurable subsets of $S \mathbb{X}$. Let $A=\bigcap_{n<\omega} \llbracket \psi_{n} \rrbracket_{S}^{\alpha}$, also measurable. Hence if $\mu \in \Delta S X$, Lemma 2.1(1) implies $\mu(A)=\lim _{n \rightarrow \infty} \mu\left(\llbracket \psi_{n} \rrbracket_{S}^{\alpha}\right)$. But then if $\alpha, \mu \models_{\Delta S}[\geqslant p]\left(\bigwedge_{\omega} \Gamma\right)$, since $\psi_{n} \in \bigwedge_{\omega} \Gamma$ we get $\alpha, \mu \models_{\Delta S}[\geqslant p] \psi_{n}$, hence $\mu\left(\llbracket \psi_{n} \rrbracket_{S}^{\alpha}\right) \geqslant p$, for all $n$. Therefore $\mu(A) \geqslant p$, because a non-increasing sequence of non-negative reals converges to its greatest lower bound. Since $\Gamma=_{S}^{\alpha} \varphi, A=\bigcap_{n<\omega} \llbracket \varphi_{n} \rrbracket_{S}^{\alpha} \subseteq \llbracket \varphi \rrbracket_{S}^{\alpha}$, implying $\mu\left(\llbracket \varphi \rrbracket_{S}^{\alpha}\right) \geqslant p$, hence $\alpha, \mu=_{\Delta S}[\geqslant p] \varphi$.

Example 4.9 Without the assumption of countability of $\Gamma$, the Countable Additivity Rule need not hold for Conseq $_{T}$. For example, let $\mathbb{X}$ be $[0,1]$ with $\mathcal{A}_{\mathbb{X}}^{g}$ being the set of open sets, generating the Borel $\sigma$-algebra, and take $T=\Delta \mathbb{X}$. Let $\Gamma=\{[0,1]-\{r\} \mid r \in[0,1]\} \subseteq \mathcal{A}_{\mathbb{X}}^{g}$. Then $\Gamma \models_{\mathbb{X}} \perp_{\mathbb{X}}$, since $\bigcap_{r}([0,1]-\{r\})=\emptyset$. But if $\mu \in \Delta \mathbb{X}$ is the standard (Lebesgue) measure, then in any $T$-coalgebra we get $\alpha, \mu \models \Delta \mathbb{X}[\geqslant 1]\left(\bigwedge_{\omega} \Gamma\right)$, since the intersection of finitely many sets of the form $[0,1]-\{r\}$ has measure 1 , while of course $\alpha, \mu \forall_{\Delta \mathbb{X}}[\geqslant 1] \perp_{\mathbb{X}}$. So this shows that Conseq $_{T}$ does not even satisfy the Almost Sure Box Rule for this uncountable $\Gamma$.

Definition 4.10 For any $S \in \operatorname{lng} T$, a set $\Gamma \subseteq$ Form $_{S}$ is:

- closed under Detachment if $\varphi, \varphi \rightarrow \psi \in \Gamma$ implies $\psi \in \Gamma$;

- an $S$-theory if it includes the set $A x_{S}$ of $S$-axioms and is closed under Detachment;

- negation complete if for every $\varphi: S$, either $\varphi \in \Gamma$ or $\neg \varphi \in \Gamma$;

- $\perp$-free if $\perp_{S} \notin \Gamma$.

Using the fact that all tautologies are axioms, together with Theorem 4.2, standard arguments show:

\section{Lemma 4.11}

(1) If $\Gamma$ is a negation complete $S$-theory, then:

$$
\begin{aligned}
& \varphi \vee \psi \in \Gamma \quad \text { iff } \quad \varphi \in \Gamma \text { or } \psi \in \Gamma \\
& \varphi \wedge \psi \in \Gamma \quad \text { iff } \quad \varphi \in \Gamma \text { and } \psi \in \Gamma \\
& \varphi \rightarrow \psi \in \Gamma \quad \text { iff } \quad \varphi \in \Gamma \text { implies } \psi \in \Gamma \\
& \varphi \leftrightarrow \psi \in \Gamma \quad \text { iff } \quad(\varphi \in \Gamma \text { iff } \psi \in \Gamma), \\
& \text { and if } \Gamma \text { is } \perp \text {-free, then } \\
& \neg \varphi \in \Gamma \quad \text { iff } \quad \varphi \notin \Gamma .
\end{aligned}
$$


(2) Every $S$-description set $\operatorname{des}_{S}^{\alpha}(x)$ is a negation complete $\perp$-free $S$-theory.

The property "negation complete $\perp$-free $S$-theory" is too weak to characterise the description sets. For that we first need to consider notions of deductive consistency:

Definition 4.12 Let $\left\{\vdash_{S} \mid S \in \operatorname{lng} T\right\}$ be any $T$-deduction system. A set $\Gamma: S$ of formulas is

- $\vdash_{S}$-inconsistent if $\Gamma \vdash_{S} \perp_{S}$, and $\vdash_{S}$-consistent otherwise;

- finitely $\vdash_{S}$-consistent if all finite subsets of $\Gamma$ are $\vdash_{S}$-consistent;

- maximally finitely $\vdash_{S}$-consistent if it is finitely $\vdash_{S}$-consistent but has no proper extension that is finitely $\vdash_{S}$-consistent;

- maximally $\vdash_{S}$-consistent if it is $\vdash_{S}$-consistent but has no proper extension that is $\vdash_{S^{-}}$ consistent;

- $\vdash_{S}$-maximal if it is negation complete and $\vdash_{S}$-consistent.

Notice that for the semantic consequence system $\operatorname{Conseq}_{T}^{\alpha}$ of a coalgebra $\alpha$ (see Theorem 4.8(1)), $\models_{S}^{\alpha}$-consistency of $\Gamma$, i.e. $\Gamma \nvdash_{S}^{\alpha} \perp_{S}$, just means satisfiability of $\Gamma$ at some state of $\alpha$. For the global consequence system Conseq $_{T},=_{S}$-consistency means satisfiability in some coalgebra.

Here are some standard relationships between the notions of Definition 4.12:

\section{Lemma 4.13}

(1) If $\Gamma \vdash_{S} \varphi$ and $\Gamma \vdash_{S} \neg \varphi$, then $\Gamma$ is $\vdash_{S}$-inconsistent.

(2) If $\Gamma$ is finitely $\vdash_{S}$-consistent, then so is one of $\Gamma \cup\{\varphi\}$ and $\Gamma \cup\{\neg \varphi\}$ for each $\varphi$.

(3) If $\Gamma$ is negation complete and finitely $\vdash_{S}$-consistent, then it is an $S$-theory.

(4) $\Gamma$ is maximally finitely $\vdash_{S}$-consistent iff it is negation complete and finitely $\vdash_{S}$-consistent.

(5) $\Gamma$ is $\vdash_{S}$-maximal iff it is maximally $\vdash_{S}$-consistent.

Proof. For (1)-(4) see [Gol93]. For (5), if $\Gamma$ is $\vdash_{S}$-maximal, then it is $\vdash_{S}$-consistent, hence finitely $\vdash_{S}$-consistent by Monotonicity, and negation complete, so altogether maximally finitely $\vdash_{S}$-consistent by result (4). But then if it has no finitely $\vdash_{S}$-consistent proper extensions, it can have no $\vdash_{S}$-consistent ones.

Conversely, if $\Gamma$ is maximally $\vdash_{S}$-consistent, it suffices to show that it is negation complete. But if both $\varphi \notin \Gamma$ and $\neg \varphi \notin \Gamma$, then $\Gamma \cup\{\varphi\} \vdash_{S} \perp_{S}$ and $\Gamma \cup\{\neg \varphi\} \vdash_{S} \perp_{S}$ by maximal consistency of $\Gamma$, hence $\Gamma \vdash_{S} \neg \varphi$ and $\Gamma \vdash_{S} \neg \neg \varphi$ by the Deduction Rule. But by (1), this contradicts the $\vdash_{S}$-consistency of $\Gamma$.

Corollary 4.14 If $\Gamma$ is $\vdash_{S}$-maximal, then:

(1) $\Gamma$ is $\vdash_{S}$-closed, i.e. if $\Sigma \subseteq \Gamma$ and $\Sigma \vdash_{S} \varphi$, then $\varphi \in \Gamma$.

(2) $\vdash_{S} \varphi$ implies $\varphi \in \Gamma$.

Proof.

(1) If $\Sigma \subseteq \Gamma$ and $\Sigma \vdash_{S} \varphi$, then $\Gamma \vdash_{S} \varphi$ by Monotonicity. But now if $\varphi \notin \Gamma$, then $\neg \varphi \in \Gamma$, so $\Gamma \vdash_{S} \neg \varphi$, contradiction the $\vdash_{S}$-consistency of $\Gamma$. 
(2) If $\emptyset \vdash_{S} \varphi$, then $\Gamma \vdash_{S} \varphi$ by Monotonicity, hence $\varphi \in \Gamma$ by (1).

We turn now to the main role of the Definite Box Rule. For each edge $S \stackrel{\kappa}{\rightsquigarrow} S^{\prime}$ with $\kappa$ definite, and each $\Gamma: S$, let

$$
[\kappa]^{-} \Gamma=\left\{\varphi: S^{\prime} \mid[\kappa] \varphi \in \Gamma\right\} .
$$

Lemma 4.15 Let $\Gamma$ be $\vdash_{S}$-maximal.

(1) If $\kappa$ is of the form $\mathrm{pr}_{j}$ or $\mathrm{ev}_{e}$ or next, then $[\kappa]^{-} \Gamma$ is $\vdash_{S^{\prime}}$-maximal.

(2) If $\kappa$ is $\mathrm{in}_{j}$, then $\neg\left[\mathrm{in}_{j}\right] \perp_{S_{j}} \in \Gamma$ implies $\left[\mathrm{in}_{j}\right]^{-} \Gamma$ is $\vdash_{S_{j}}$-maximal.

Proof. First, note that $[\kappa]^{-} \Gamma$ is always negation complete, for if $\varphi \notin[\kappa]^{-} \Gamma$, then $\neg[\kappa] \varphi \in \Gamma$ by negation completeness of $\Gamma$, and $\neg[\kappa] \varphi \rightarrow[\kappa] \neg \varphi$ is an axiom of type (a), hence in $\Gamma$, so $[\kappa] \neg \varphi \in \Gamma$, giving $\neg \varphi \in[\kappa]^{-} \Gamma$.

If remains to show that $[\kappa]^{-} \Gamma$ is $\vdash_{S^{\prime}}$-consistent. But if $[\kappa]^{-} \Gamma \vdash_{S^{\prime}} \perp_{S^{\prime}}$ then by the Definite Box Rule $\{[\kappa] \varphi \mid[\kappa] \varphi \in \Gamma\} \vdash_{S}[\kappa] \perp_{S^{\prime}}$, hence $\Gamma \vdash_{S}[\kappa] \perp_{S^{\prime}}$ by Monotonicity. If (1) $\kappa$ is $\operatorname{pr}_{j}$ or ev $_{e}$ or next, this is a contradiction, as then $\neg[\kappa] \perp_{S^{\prime}}$ is an axiom, so $\Gamma \vdash_{S} \neg[\kappa] \perp_{S^{\prime}}$ in contradiction to the consistency of $\Gamma$. For (2), it is similarly a contradiction if $\neg\left[\mathrm{in}_{j}\right] \perp_{S_{j}} \in \Gamma$.

Definition 4.16 A $T$-deduction system is

- sound if $\Gamma \vdash_{S} \varphi$ implies $\Gamma \models_{S} \varphi$, for all $S \in \operatorname{lng} T$; and

- Lindenbaum if, for all $S \in \operatorname{lng} T$, every $\vdash_{S}$-consistent set of formulas is included in some $\vdash_{S}$-maximal set.

\section{Theorem 4.17}

(1) A T-deduction system is sound iff every satisfiable set of $S$-formulas is $\vdash_{S}$-consistent.

(2) If a T-deduction system is sound, then every $S$-description set is $\vdash_{S}$-maximal.

(3) For any $T$-coalgebra $\alpha$, Conseq $_{T}^{\alpha}=\left\{\left.\right|_{S} ^{\alpha} \mid S \in \operatorname{Ing} T\right\}$ is a Lindenbaum T-deduction system.

(4) Conseq $_{T}=\left\{\models_{S} \mid S \in \operatorname{lng} T\right\}$ is a sound Lindenbaum T-deduction system.

Proof.

(1) Assume soundness. If $\Gamma: S$ is satisfiable, then $\alpha, x \models_{S} \Gamma$ for some $x$ in some $T$-coalgebra $\alpha$. Since $\alpha, x \not \forall_{S} \perp_{S}$ this gives $\Gamma \not \nvdash_{S} \perp_{S}$, so soundness implies $\Gamma \nvdash_{S} \perp$.

Conversely, assume satisfiable $S$-sets are $\vdash_{S}$-consistent. Then $\Gamma \not \nvdash_{S} \varphi$ implies $\Gamma \cup\{\neg \varphi\}$ is satisfiable, hence $\Gamma \cup\{\neg \varphi\} \forall_{S} \perp_{S}$ by assumption, so $\Gamma \nvdash_{S} \varphi$ by Lemma 4.5(5). Thus soundness holds.

(2) Given soundness, any $S$-description set is $\vdash_{S}$-consistent by (1), since it is satisfiable by definition. But every description set is negation complete.

(3) Conseq $_{T}^{\alpha}$ is a $T$-deduction system by Theorem 4.8. For the Lindenbaum property: if $\Gamma$ is $\models_{S}^{\alpha}$-consistent, i.e. is satisfiable in $\alpha$, then $\alpha, x \models_{S} \Gamma$ for some $x$. Then the description set $\operatorname{des}_{S}^{\alpha}(x)$ extends $\Gamma$ and is negation complete and $\models_{S}^{\alpha}$-consistent.

(4) Conseq $_{T}$ is sound tautologically. For the Lindenbaum property, if $\Gamma$ is $\models_{S}$-consistent, then $\alpha, x \models_{S} \Gamma$ for some $x$ in some $T$-coalgebra $\alpha$. But then the extension $\operatorname{des}_{S}^{\alpha}(x)$ of $\Gamma$ is $\models_{S^{-}}$ maximal by (2). (Alternatively, $\operatorname{des}_{S}^{\alpha}(x)$ is $\models_{S}^{\alpha}$-consistent as in (3), hence $\models_{S}$-consistent as $\models_{S}$ is included in $\models_{S}^{\alpha}$.) 
As usual, one of the important roles of the Lindenbaum property is to ensure

Lemma 4.18 In a Lindenbaum deduction system, $\vdash_{S} \varphi$ if, and only if $\varphi$ belongs to every $\vdash_{S^{-}}$ maximal set.

Proof. From left to right is by Corollary 4.14(2) But if $\nvdash_{S} \varphi$, then $\{\neg \varphi\} \nvdash_{S} \perp_{S}$ by Lemma 4.5(5), so by the Lindenbaum property there is a $\vdash_{S}$-maximal $\Gamma$ extending $\{\neg \varphi\}$, and hence $\varphi \notin \Gamma$ by $\vdash_{S^{-}}$consistency of $\Gamma$.

\section{Canonical Spaces and Coalgebras}

We are going to show that the system Conseq $_{T}$ of satisfaction relations $\models_{S}$ is the least Lindenbaum deduction system for $T$, and the only one that is sound. To do this we fix a Lindenbaum $T$-deduction system $D=\left\{\vdash_{S} \mid S \in \operatorname{Ing} T\right\}$ and build a $T$-coalgebra $\left(\mathbb{X}^{D}, \alpha^{D}\right)$ such that the $\vdash_{S}$-maximal sets correspond to the points of the space $S \mathbb{X}^{D}$ (for their precise relationship, see Lemma 5.7 and Theorem 5.11). From this we conclude that all $\vdash_{S}$-maximal sets are $S$-description sets, conversely to Theorem 4.17(2). The idea of this construction was developed algebraically in [Jac01] for functors over Set, and adapted in [MV04, Vig05, MV06] to a model-theoretic construction of final coalgebras for functors over Meas, working directly with description sets. Here we give a proof-theoretic version, following the methodology of Sections 3 and 4 of [MV06], but working instead with the maximal sets.

\section{Definition 5.1}

- $X_{S}^{D}=\left\{\Gamma \subseteq\right.$ Form $_{S} \mid \Gamma$ is $\vdash_{S}$-maximal $\}$

- $|\varphi|_{S}=\left\{x \in X_{S}^{D} \mid \varphi \in x\right\}$

- $\mathcal{A}_{S}^{D}=\left\{|\varphi|_{S} \mid \varphi:: S\right\}$

- $\mathbb{X}_{S}^{D}=\left(X_{S}^{D}, \sigma\left(\mathcal{A}_{S}^{D}\right)\right)$.

The facts in Lemma 4.11(1) ensure that the Boolean connectives are interpreted under $\varphi \mapsto|\varphi|_{S}$ as the Boolean set operations, i.e. $|\neg \varphi|_{S}=X_{S}^{D}-|\varphi|_{S},|\varphi \vee \psi|_{S}=|\varphi|_{S} \cup|\psi|_{S}$ etc. Thus $\mathcal{A}_{S}^{D}$ is an algebra of sets and $\mathbb{X}_{S}^{D}$ the measurable space it generates.

We will need the Lindenbaum property of $D$ in a number of places, including to show

Lemma 5.2 For any $\varphi, \psi: S$,

(1) $|\varphi|_{S} \subseteq|\psi|_{S}$ iff $\vdash_{S} \varphi \rightarrow \psi$.

(2) $|\varphi|_{S}=|\psi|_{S}$ iff $\vdash_{S} \varphi \leftrightarrow \psi$.

Proof. $\quad$ For (1): by Lemma 4.18, $\vdash_{S} \varphi \rightarrow \psi$ iff $\varphi \rightarrow \psi$ belongs to every member of $X_{S}^{D}$, which by Lemma 4.11 is equivalent to requiring that every member of $X_{S}^{D}$ containing $\varphi$ must also contain $\psi$.

(2) follows from (1) by Boolean reasoning.

\section{Definition 5.3 (Canonical measures)}

If $\Delta S \in \operatorname{lng} T$, for each $\vdash_{S}$-maximal set $x \in X_{\Delta S}^{D}$, define a function $\mu_{x}$ on $\mathcal{A}_{S}^{D}$ by

$$
\mu_{x}\left(|\varphi|_{S}\right)=\sup \left\{q \in[0,1]_{\mathbb{Q}} \mid[\geqslant q] \varphi \in x\right\} .
$$


Theorem $\mathbf{5 . 4} \mu_{x}$ is a well-defined probability measure on $\mathcal{A}_{S}^{D}$.

Proof. $\quad$ First observe that if $|\varphi|_{S}=|\psi|_{S}$, then $\vdash_{S} \varphi \leftrightarrow \psi$, hence $\vdash_{\Delta S}[\geqslant q] \varphi \leftrightarrow[\geqslant q] \psi$ for any $q$ by Lemma $4.6(6)$, so $[\geqslant q] \varphi \in x$ iff $[\geqslant q] \psi \in x$. Thus the definition of $\mu_{x}\left(|\varphi|_{S}\right)$ does not depend on how $|\varphi|_{S}$ is named.

Now Lemma 4.6(1) implies that $0 \in\{q:[\geqslant q] \varphi \in x\}$, so $\mu_{x}\left(|\varphi|_{S}\right)$ does exist as a supremum, and we get $\mu_{x}: X_{S}^{D} \rightarrow[0,1]$. Moreover, as $\emptyset=\left|\perp_{S}\right|$, and Lemma 4.6(2) guarantees that $[\geqslant q] \perp_{S} \notin x$ if $q>0$, we have $\mu_{x} \emptyset=0$.

We will use the fact that for any $\varphi:: S$ and $p \in[0,1]_{Q}$,

$$
\mu_{x}\left(|\varphi|_{S}\right)>p \quad \text { implies } \quad[\geqslant p] \varphi \in x .
$$

To see why: if $\mu_{x}\left(|\varphi|_{S}\right)>p$ but $[\geqslant p] \varphi \notin x$, then Lemma 4.6(7) ensures that $[\geqslant q] \varphi \notin x$ for any rational $q>p$, hence $[\geqslant q] \varphi \in x$ only if $q \leqslant p$. However this contradicts the definition of $\mu_{x}\left(|\varphi|_{S}\right)$ as a supremum. Hence (5.1) holds.

Next we show that

$$
\mu_{x}\left(\left|\varphi_{1}\right|_{S}\right)+\mu_{x}\left(\left|\varphi_{2}\right|_{S}\right) \geqslant \mu_{x}\left(\left|\varphi_{1}\right|_{S} \cup\left|\varphi_{2}\right|_{S}\right) .
$$

If this were false, we could choose rationals $q_{1}, q_{2}$ with

$$
\mu_{x}\left(\left|\varphi_{1}\right|_{S}\right)+\mu_{x}\left(\left|\varphi_{2}\right|_{S}\right)<q_{1}+q_{2}<\mu_{x}\left(\left|\varphi_{1}\right|_{S} \cup\left|\varphi_{2}\right|_{S}\right)=\mu_{x}\left(\left|\varphi_{1} \vee \varphi_{2}\right|_{S}\right) \leqslant 1
$$

and $\mu_{x}\left(\left|\varphi_{i}\right|_{S}\right)<q_{i}$ for $i=1,2$. But then $\left[\geqslant q_{i}\right] \varphi_{i} \notin x$ by definition of $\mu_{x}\left(\left|\varphi_{i}\right|_{S}\right)$, hence $\neg\left[\geqslant q_{i}\right] \varphi_{i} \in$ $x$. By invoking axiom (7e) we infer from this that $\left[\geqslant q_{1}+q_{2}\right]\left(\varphi_{1} \vee \varphi_{2}\right) \notin x$. But then by (5.1), $\mu_{x}\left(\left|\varphi_{1} \vee \varphi_{2}\right|_{S}\right) \ngtr q_{1}+q_{2}$, a contradiction. So (5.2) is true.

The next step is to show that $\mu_{x}$ is finitely additive, by proving the reverse inequality

$$
\mu_{x}\left(\left|\varphi_{1}\right|_{S}\right)+\mu_{x}\left(\left|\varphi_{2}\right|_{S}\right) \leqslant \mu_{x}\left(\left|\varphi_{1} \vee \varphi_{2}\right|_{S}\right)
$$

when $\left|\varphi_{1}\right|_{S}$ and $\left|\varphi_{2}\right|_{S}$ are disjoint, or equivalently when $\vdash_{S} \neg\left(\varphi_{1} \wedge \varphi_{2}\right)$. If (5.3) failed in this case, we could choose rationals $q_{i}<\mu_{x}\left(\left|\varphi_{i}\right|_{S}\right)$ with

$$
\mu_{x}\left(\left|\varphi_{1} \vee \varphi_{2}\right|_{S}\right)<q_{1}+q_{2}<\mu_{x}\left(\left|\varphi_{1}\right|_{S}\right)+\mu_{x}\left(\left|\varphi_{2}\right|_{S}\right) .
$$

Then (5.1) gives $\left[\geqslant q_{1}\right] \varphi_{1},\left[\geqslant q_{2}\right] \varphi_{2} \in x$. Also $q_{1}+q_{2} \leqslant 1$, for if $q_{1}+q_{2}>1$, Lemma 4.7(1) gives $\vdash_{\Delta S}\left[\geqslant q_{1}\right] \varphi_{1} \rightarrow \neg\left[\geqslant q_{2}\right] \varphi_{2}$, hence the contradictory $\neg\left[\geqslant q_{2}\right] \varphi_{2} \in x$. Thus we can apply Lemma $4.7(2)$ to infer that $\left[\geqslant q_{1}+q_{s}\right]\left(\varphi_{1} \vee \varphi_{2}\right) \in x$. But this implies the contradictory $\mu_{x}\left(\left|\varphi_{1} \vee \varphi_{2}\right|_{S}\right) \geqslant$ $q_{1}+q_{2}$ by definition of $\mu_{x}$.

That completes the proof that $\mu_{x}$ is finitely additive. To prove that it is a measure, it suffices by Lemma 2.1(2) to show that if $\left\{\left|\varphi_{n}\right|_{S} \mid n<\omega\right\}$ is a non-increasing sequence of members of $\mathcal{A}_{S}^{D}$ whose intersection is empty, then $\mu_{x}\left(\left|\varphi_{n}\right|_{S}\right) \rightarrow 0$. Now by finite additivity, the number-sequence $\left\{\mu_{x}\left(\left|\varphi_{n}\right|_{S}\right) \mid n<\omega\right\}$ is non-increasing. Since it is bounded below by 0 , it must converge to its greatest lower bound. If this limit was positive, then there would exist a rational $p$ with $\mu_{x}\left(\left|\varphi_{n}\right|_{S}\right)>p>0$ for all $n$. Then if $\Gamma=\left\{\varphi_{n} \mid n<\omega\right\}$, any $\psi \in \bigwedge_{\omega} \Gamma$ has $|\psi|_{S}=\left|\varphi_{m}\right|_{S}$ for some $m$, since the $\left|\varphi_{n}\right|_{S}$ 's are non-increasing, hence $\mu_{x}\left(|\psi|_{S}\right)>p$, so $[\geqslant p] \psi \in x$ by (5.1). This shows that $[\geqslant p]\left(\bigwedge_{\omega} \Gamma\right)$ is a subset of $x$, and therefore is $\vdash_{\Delta S}$-consistent by the consistency of $x$. But $\vdash_{\Delta S} \neg[\geqslant p] \perp_{S}$ by Lemma $4.6(2)$, so this implies $[\geqslant p]\left(\bigwedge_{\omega} \Gamma\right) \forall \Delta_{S}[\geqslant p] \perp_{S}$. By the Countable Additivity Rule it then follows that $\Gamma \nvdash_{S} \perp_{S}$, so by the Lindenbaum property, there is some $y \in X_{S}^{D}$ with $\Gamma \subseteq y$, hence $y \in \bigcap_{n<\omega}\left|\varphi_{n}\right|_{S}$, contradicting the fact that this intersection is empty.

Hence $\lim _{n \rightarrow \infty} \mu_{x}\left(\left|\varphi_{n}\right|_{S}\right)=0$ as required.

That was the only use we need to make of the Countable Additivity Rule, and the argument given shows that we really only needed its special case 


$$
\Gamma \vdash_{S} \perp_{S} \quad \text { implies } \quad[\geqslant p]\left(\bigwedge_{\omega} \Gamma\right) \vdash_{\Delta S} \perp_{\Delta S} \text {. }
$$

Here is where the Archimedean Rule is needed:

Lemma 5.5 For all $|\varphi|_{S} \in \mathcal{A}_{S}^{D}$ and $p \in[0,1]_{\mathbb{Q}}$,

$$
\mu_{x}\left(|\varphi|_{S}\right) \geqslant p \quad \text { iff } \quad[\geqslant p] \varphi \in x
$$

Proof. From right to left holds by definition of $\mu_{x}$. Conversely, if $\mu_{x}\left(|\varphi|_{S}\right) \geqslant p$, then for all $q<p$ we get $[\geqslant q] \varphi \in x$ by (5.1). The Archimedean Rule and the $\vdash_{\Delta S}$-closure of $x$ (Corollary $4.14(1))$ then ensure that $[\geqslant p] \varphi \in x$.

Of course Lemma 5.5 implies (5.1), but we deferred it till after the proof of Theorem 5.4 in order to make clear that the Archimedean Rule is not needed to prove that $\mu_{x}$ is a measure.

To increase legibility, we may drop the $D$-superscripts in referring to the spaces $\mathbb{X}_{S}^{D}$, understanding that the definition of $\mathbb{X}_{S}$ depends on a particular deduction system.

Lemma 5.6 There exist measurable functions

$$
\begin{aligned}
\mathbb{X}_{S_{1} \times S_{2}} & \stackrel{\rho_{S_{1} \times S_{2}}}{\longrightarrow} \mathbb{X}_{S_{1}} \times \mathbb{X}_{S_{2}} \\
\mathbb{X}_{S_{1}+S_{2}} & \stackrel{\rho_{S_{1}+S_{2}}}{\longrightarrow} \mathbb{X}_{S_{1}}+\mathbb{X}_{S_{2}} \\
\mathbb{X}_{S^{E}} & \stackrel{\rho_{S}}{\longrightarrow}\left(\mathbb{X}_{S}\right)^{E} \\
\mathbb{X}_{I d} & \stackrel{\rho_{I d}}{\longrightarrow} \mathbb{X}_{T} \\
\mathbb{X}_{\Delta S} & \stackrel{\rho_{\Delta S}}{\longrightarrow} \Delta\left(\mathbb{X}_{S}\right)
\end{aligned}
$$

such that

$$
\begin{aligned}
\rho_{S_{1} \times S_{2}}^{-1}\left(\left|\varphi_{1}\right|_{S_{1}} \times\left|\varphi_{2}\right|_{S_{2}}\right) & =\left|\left[\mathrm{pr}_{1}\right] \varphi_{1} \wedge\left[\mathrm{pr}_{2}\right] \varphi_{2}\right|_{S_{1} \times S_{2}} \\
\rho_{S_{1}+S_{2}}^{-1}\left(\operatorname{in}_{j}\left(|\varphi|_{S_{j}}\right)\right) & =\left|\neg\left[\mathrm{in}_{j}\right] \perp_{S_{j}} \wedge\left[\mathrm{in}_{j}\right] \varphi\right|_{S_{1}+S_{2}} \\
\rho_{S^{E}}^{-1}\left(e v_{e}^{-1}|\varphi|_{S}\right) & =\left|\left[\mathrm{ev}_{e}\right] \varphi\right|_{S^{E}} \\
\rho_{I d}^{-1}\left(|\varphi|_{T}\right) & =\mid\left.[\text { next }] \varphi\right|_{I d} \\
\rho_{\Delta S}^{-1}\left(\beta^{p}\left(|\varphi|_{S}\right)\right) & =|[\geqslant p] \varphi|_{\Delta S} .
\end{aligned}
$$

Proof. It suffices to define functions $\rho$ satisfying the stated equations, since their measurability immediately follows. This is because each equation has the form $\rho^{-1}(A)=B$, such that when $A$ is any generator of the $\sigma$-algebra of the codomain of $\rho$, then $B$ is a measurable set in the domain space of $\rho$ (recall that the $\sigma$-algebra of $\mathbb{X}_{S}$ is generated by the sets $|\varphi|_{S}$ with $\varphi:: S$ ).

- Let $\rho_{S_{1} \times S_{2}}(x)=\left(\left[\mathrm{pr}_{1}\right]^{-} x,\left[\mathrm{pr}_{2}\right]^{-} x\right)$ for any $\vdash_{S_{1} \times S_{2}}$-maximal set $x$. Here $\left[\mathrm{pr}_{j}\right]^{-} x$ is the $S_{j}$-maximal set $\left\{\varphi: S_{j} \mid\left[\operatorname{pr}_{j}\right] \varphi \in x\right\}$ given by Lemma $4.15(1)$. For $(5.4), \rho_{S_{1} \times S_{2}}(x) \in$ $\left|\varphi_{1}\right|_{S_{1}} \times\left|\varphi_{2}\right|_{S_{2}}$ iff $\varphi_{1} \in\left[\mathrm{pr}_{1}\right]^{-} x$ and $\varphi_{2} \in\left[\mathrm{pr}_{2}\right]^{-} x$, iff $\left[\mathrm{pr}_{1}\right] \varphi_{1} \wedge\left[\mathrm{pr}_{2}\right] \varphi_{2} \in x$.

- $\rho_{S_{1}+S_{2}}$ is defined as follows. For each $\vdash_{S_{1}+S_{2}}$-maximal $x$, by axiom (4b) there is exactly one $j \in\{1,2\}$ such that $\neg\left[\operatorname{in}_{j}\right] \perp_{S_{j}} \in x$. Put $\rho_{S_{1}+S_{2}}(x)=i n_{j}\left(\left[\operatorname{in}_{j}\right]^{-} x\right)$ for this $j$, noting that $\left[\mathrm{in}_{j}\right]^{-} x$ itself belongs to $\mathbb{X}_{S_{j}}$ by Lemma 4.15(2). Hence for each $j \in\{1,2\}$ we have $\rho_{S_{1}+S_{2}}(x) \in i n_{j}\left(X_{S_{j}}\right)$ iff $\neg\left[\operatorname{in}_{j}\right] \perp_{S_{j}} \in x$. 
To prove (5.5), for all $x \in X_{S_{1}+S_{2}}$ and $\varphi: S_{j}$ we have

$$
\begin{aligned}
& \rho_{S_{1}+S_{2}}(x) \in i n_{j}\left(|\varphi|_{S_{j}}\right) \\
& \text { iff } \neg\left[\mathrm{in}_{j}\right] \perp_{S_{j}} \in x \text { and } i n_{j}\left(\left[\mathrm{in}_{j}\right]^{-} x\right) \in i n_{j}\left(|\varphi|_{S_{j}}\right) \\
& \text { iff } \neg\left[\mathrm{in}_{j}\right] \perp_{S_{j}} \in x \text { and }\left[\mathrm{in}_{j}\right]^{-} x \in|\varphi|_{S_{j}} \quad \text { as } i n_{j} \text { is injective } \\
& \text { iff } \neg\left[\mathrm{in}_{j}\right] \perp_{S_{j}} \in x \text { and }\left[\mathrm{in}_{j}\right] \varphi \in x \\
& \text { iff }\left(\neg\left[\operatorname{in}_{j}\right] \perp_{S_{j}} \wedge\left[\mathrm{in}_{j}\right] \varphi\right) \in x .
\end{aligned}
$$

- Let $\rho_{S^{E}}(x)$ be the function $E \rightarrow X_{S}$ defined by $\rho_{S^{E}}(x)(e)=\left[\mathrm{ev}_{e}\right]^{-} x$, which is $\vdash_{S^{-} \text {maximal }}$ by Lemma 4.15(1).

Then $x \in \rho_{S^{E}}^{-1}\left(e v_{e}^{-1}|\varphi|_{S}\right)$ iff $\rho_{S^{E}}(x)(e) \in|\varphi|_{S}$ iff $\varphi \in\left[\mathrm{ev}_{e}\right]^{-} x$ iff $\left[\mathrm{ev}_{e}\right] \varphi \in x$, giving (5.6).

- Let $\rho_{I d}(x)=[\text { next }]^{-} x$. Then (5.7) amounts to the claim that $[\text { next }]^{-} x \in|\varphi|_{T}$ iff $[$ next $] \varphi \in x$, which is true.

- $\rho_{\Delta S}$ is defined as follows. For each $x$ in $\mathbb{X}_{\Delta S}$ the function $\mu_{x}$ of Theorem 5.4 is a probability measure on the algebra $\mathcal{A}_{S}$, so has a unique extension to a probability measure on the $\sigma$-algebra generated by $\mathcal{A}_{S}$ (by the famous Carathéodory outer measure construction). We take $\rho_{\Delta S}(x)$ to be this unique extension, which will also be called $\mu_{x}$.

For (5.8), with the help of Lemma 5.5 we reason that $\rho_{\Delta S}(x) \in \beta^{p}\left(|\varphi|_{S}\right)$ iff $\mu_{x}\left(|\varphi|_{S}\right) \geqslant p$ iff $[\geqslant p] \varphi \in x$ iff $x \in|[\geqslant p] \varphi|_{\Delta S}$.

Next we introduce "definable" subsets $\|\varphi\|_{S}$ of $S\left(\mathbb{X}_{I d}\right)$, for $\varphi: S$, that correspond to the subsets $|\varphi|_{S}$ of $\mathbb{X}_{S}$. The inductive definition of $\|\varphi\|_{S}$ imitates that of $\llbracket \varphi \rrbracket_{S}^{\alpha}$, except for the formulas $[$ next $] \varphi$ :

$$
\begin{aligned}
\left\|\perp_{S}\right\|_{S} & =\emptyset \\
\|A\|_{\mathbb{X}} & =A \\
\left\|\varphi_{1} \rightarrow \varphi_{2}\right\|_{S} & =\left\|\varphi_{1}\right\|_{S} \Rightarrow\left\|\varphi_{2}\right\|_{S} \\
\left\|\left[\mathrm{pr}_{j}\right] \varphi\right\|_{S_{1} \times S_{2}} & =\pi_{j}^{-1}\|\varphi\|_{S_{j}} \\
\left\|\left[\mathrm{in}_{1}\right] \varphi\right\|_{S_{1}+S_{2}} & =i n_{1}\left(\|\varphi\|_{S_{1}}\right) \cup i n_{2}\left(S_{2} \mathbb{X}_{I d}\right) \\
\left\|\left[\mathrm{in}_{2}\right] \varphi\right\|_{S_{1}+S_{2}} & =i n_{1}\left(S_{1} \mathbb{X}_{I d}\right) \cup i n_{2}\left(\|\varphi\|_{S_{2}}\right) \\
\left\|\left[\mathrm{ev}_{e}\right] \varphi\right\|_{S^{E}} & =e v_{e}^{-1}\|\varphi\|_{S} \\
\|\left[\mathrm{next}_{\varphi} \|_{I d}\right. & =|[\mathrm{next}] \varphi|_{I d} \\
\|[\geqslant p] \varphi\|_{\Delta S} & =\beta^{p}\|\varphi\|_{S} .
\end{aligned}
$$

It follows by induction that for all $\varphi: I d,\|\varphi\|_{I d}=|\varphi|_{I d} \subseteq X_{I d}$ (see Lemma 4.11(1)).

Lemma 5.7 For each $S \in \operatorname{Ing} T$ there is a measurable map $\mathrm{r}_{S}: \mathbb{X}_{S} \rightarrow S\left(\mathbb{X}_{I d}\right)$ such that for all formulas $\varphi: S$ we have

$$
\mathrm{r}_{S}^{-1}\|\varphi\|_{S}=|\varphi|_{S}, \quad \text { i.e. } \mathrm{r}_{S}(x) \in\|\varphi\|_{S} \text { iff } \varphi \in x .
$$

Proof. By induction on the formation of $S$. The class of $S$-formulas satisfying (5.9) is readily seen to be closed under the Boolean connectives: this does not need to be repeated for each case of $S$. The cases are as follows. 
- $S=I d$ : let $\mathrm{r}_{I d}$ be the identity function on $\mathbb{X}_{I d}$. Measurability is immediate and (5.9) just repeats that $\|\varphi\|_{I d}=|\varphi|_{I d}$.

- $S=\mathbb{X}$, the functor with constant value $\mathbb{X}$. Here $r_{\mathbb{X}}: X_{\mathbb{X}} \rightarrow \mathbb{X}$ is defined by: $r_{\mathbb{X}}(x)=$ the unique $c \in X$ such that $\{c\} \in x$. This exists because the Constant Rule and $\vdash_{\mathbb{X}}$-consistency of $x$ ensure that we cannot have every $c \in X$ satisfying $\neg\{c\} \in x$, so $\{c\} \in x$ for at least one $c$. But by axiom (2b), if $c \neq d \in X$, then $(\{c\} \rightarrow \neg\{d\}) \in x$, so if $\{c\} \in x$ then $\{d\} \notin x$.

Thus $\left\{r_{\mathbb{X}}(x)\right\} \in x$. Now axioms $(2 \mathrm{a})$ and $(2 \mathrm{~b})$ give, for all $A: \mathbb{X}$, that $\left(\left\{r_{\mathbb{X}}(x)\right\} \rightarrow A\right) \in x$ if $r_{\mathbb{X}}(x) \in A$ and $\left(\left\{r_{\mathbb{X}}(x)\right\} \rightarrow \neg A\right) \in x$ if $\mathbf{r}_{\mathbb{X}}(x) \notin A$. This implies that $r_{\mathbb{X}}(x) \in A$ iff $A \in x$, which is (5.9), and that $r_{\mathbb{X}}^{-1}(A)=|A|_{\mathbb{X}}$. In particular this holds for the generators $A \in \mathcal{A}_{\mathbb{X}}^{g}$ of the measure algebra of the constant space $\mathbb{X}$, which is enough to make $\mathbf{r}_{\mathbb{X}}$ a measurable function.

- $S=S_{1} \times S_{2}$ : assume that $\mathrm{r}_{S_{1}}$ and $\mathrm{r}_{S_{1}}$ are defined and fulfil the Lemma. Let $\mathrm{r}_{S_{1} \times S_{2}}$ be the composition of

$$
\mathbb{X}_{S_{1} \times S_{2}} \stackrel{\rho_{S_{1} \times S_{2}}}{\longrightarrow} \mathbb{X}_{S_{1}} \times \mathbb{X}_{S_{2}} \stackrel{\mathrm{r}_{S_{1}} \times \mathrm{r}_{S_{2}}}{\longrightarrow} S_{1}\left(\mathbb{X}_{I d}\right) \times S_{2}\left(\mathbb{X}_{I d}\right)
$$

Since $r_{S_{1}}$ and $r_{S_{2}}$ are measurable, so too is their product $r_{S_{1}} \times r_{S_{2}}(2.2)$, and hence so too is its composition with the measurable $\rho_{S_{1} \times S_{2}}$.

For (5.9), the definitions of the operators $\rho$ and $r$ give

$$
\mathrm{r}_{S_{1} \times S_{2}}(x)=\left(\mathrm{r}_{S_{1}}\left(\left[\mathrm{pr}_{1}\right]^{-} x\right), \mathrm{r}_{S_{2}}\left(\left[\mathrm{pr}_{2}\right]^{-} x\right)\right),
$$

so $\mathrm{r}_{S_{1} \times S_{2}}(x) \in\left\|\left[\mathrm{pr}_{j}\right] \varphi\right\|_{S_{1} \times S_{2}}=\pi_{j}^{-1}\|\varphi\|_{S_{j}}$ iff $\mathrm{r}_{S_{j}}\left(\left[\mathrm{pr}_{j}\right]^{-} x\right) \in\|\varphi\|_{S_{j}}$ iff $\varphi \in\left[\mathrm{pr}_{j}\right]^{-} x$ (by induction hypothesis on $\mathrm{r}_{S_{j}}$ ), iff $\left[\mathrm{pr}_{j}\right] \varphi \in x$.

- $S=S_{1}+S_{2}$ : let $\mathrm{r}_{S_{1}+S_{2}}$ be the composition of the measurable functions

$$
\mathbb{X}_{S_{1}+S_{2}} \stackrel{\rho_{S_{1}+S_{2}}}{\longrightarrow} \mathbb{X}_{S_{1}}+\mathbb{X}_{S_{2}} \stackrel{\mathrm{r}_{S_{1}}+\mathrm{r}_{S_{2}}}{\longrightarrow} S_{1}\left(\mathbb{X}_{I d}\right)+S_{2}\left(\mathbb{X}_{I d}\right)
$$

Thus

$$
\mathrm{r}_{S_{1}+S_{2}}(x)=i n_{j}\left(\mathrm{r}_{S_{j}}\left(\left[\mathrm{in}_{j}\right]^{-} x\right)\right),
$$

for the unique $j$ such that $\neg\left[\mathrm{in}_{j}\right] \perp_{S_{j}} \in x$.

Now $\left(\mathrm{r}_{S_{1}+S_{2}}\right)^{-1}$ is $\rho_{S_{1}+S_{2}}^{-1} \circ\left(\mathrm{r}_{S_{1}}+\mathrm{r}_{S_{2}}\right)^{-1}$. Take a formula of type [in 1$] \varphi$. Since $\left\|\left[\mathrm{in}_{1}\right] \varphi\right\|_{S_{1}+S_{2}}$ is the union of the two sets $i n_{1}\left(\|\varphi\|_{S_{1}}\right)$ and $i n_{2}\left(S_{2} \mathbb{X}_{I d}\right)$ from the disjoint components of $S_{1}\left(\mathbb{X}_{I d}\right)+S_{2}\left(\mathbb{X}_{I d}\right)$, we have

$$
\left(\mathrm{r}_{S_{1}}+\mathrm{r}_{S_{2}}\right)^{-1}\left\|\left[i \mathrm{n}_{1}\right] \varphi\right\|_{S_{1}+S_{2}}=i n_{1}\left(\mathrm{r}_{S_{1}}^{-1}\|\varphi\|_{S_{1}}\right) \cup i n_{2}\left(X_{S_{2}}\right),
$$

which is equal to $i n_{1}\left(|\varphi|_{S_{1}}\right) \cup i n_{2}\left(X_{S_{2}}\right)$ by hypothesis on $r_{S_{1}}$. Hence $r_{S_{1}+S_{2}}^{-1}\left\|\left[i n_{1}\right] \varphi\right\|_{S_{1}+S_{2}}$ is

$$
\left(\rho_{S_{1}+S_{2}}\right)^{-1} i n_{1}\left(|\varphi|_{S_{1}}\right) \cup\left(\rho_{S_{1}+S_{2}}\right)^{-1} i n_{2}\left(X_{S_{2}}\right),
$$

which, by (5.5) and the definition of $\rho_{S_{1}+S_{2}}$, is

$$
\begin{aligned}
& \left|\neg\left[\mathrm{in}_{1}\right] \perp_{S_{1}} \wedge\left[\mathrm{in}_{1}\right] \varphi\right|_{S_{1}+S_{2}} \cup\left|\left[\mathrm{in}_{1}\right] \perp_{S_{1}}\right|_{S_{1}+S_{2}} \\
& =\left|\left[\mathrm{in}_{1}\right] \varphi\right|_{S_{1}+S_{2}} \cup\left|\left[\mathrm{in}_{1}\right] \perp_{S_{1}}\right|_{S_{1}+S_{2}} \quad \text { by Boolean reasoning } \\
& =\left|\left[\mathrm{in}_{1}\right] \varphi\right|_{S_{1}+S_{2}},
\end{aligned}
$$

as $\vdash_{S_{1}+S_{2}}\left[\mathrm{in}_{1}\right] \perp_{S_{1}} \rightarrow\left[\mathrm{in}_{1}\right] \varphi$ by Lemma $4.6(5)$.

The proof that $\mathrm{r}_{S_{1}+S_{2}}^{-1}\left\|\left[\mathrm{in}_{2}\right] \varphi\right\|_{S_{1}+S_{2}}=\left|\left[\mathrm{in}_{2}\right] \varphi\right|_{S_{1}+S_{2}}$ is likewise, and (5.9) follows for this case. 
- Suppose $S^{E} \in \operatorname{lng} T$ and assume that $\mathrm{r}_{S}$ is defined and fulills the Lemma. Let $\mathrm{r}_{S^{E}}=$ $\mathrm{r}_{S}^{E} \circ \rho_{S^{E}}$ :

$$
\mathbb{X}_{S^{E}} \stackrel{\rho_{S}}{\longrightarrow}\left(\mathbb{X}_{S}\right)^{E} \stackrel{\mathrm{r}_{S}^{E}}{\longrightarrow}\left(S\left(\mathbb{X}_{I d}\right)\right)^{E}
$$

Then $\mathrm{r}_{S^{E}}(x)=\mathrm{r}_{S} \circ\left(\rho_{S^{E}}(x)\right)$ is the function with domain $E$ for which

$$
\mathrm{r}_{S^{E}}(x)(e)=\mathrm{r}_{S}\left(\left[\mathrm{ev}_{e}\right]^{-} x\right) .
$$

Hence $\mathrm{r}_{S^{E}}(x) \in\left\|\left[\mathrm{ev}_{e}\right] \varphi\right\|_{S^{E}}=e v_{e}^{-1}\|\varphi\|_{S}$ iff $\mathrm{r}_{S}\left(\left[\mathrm{ev}_{e}\right]^{-} x\right) \in\|\varphi\|_{S}$ iff $\varphi \in\left[\mathrm{ev}_{e}\right]^{-} x$ (by induction hypothesis on $\mathrm{r}_{S}$ ), iff $\left[\mathrm{ev}_{e}\right] \varphi \in x$, proving (5.9) in this case.

- If $\Delta S \in \operatorname{lng} T$, assume the construction of $\mathrm{r}_{S}$, and define $\mathrm{r}_{\Delta S}=\Delta \mathrm{r}_{S} \circ \rho_{\Delta S}$ :

$$
\mathbb{X}_{\Delta S} \stackrel{\rho_{\Delta S}}{\longrightarrow} \Delta\left(\mathbb{X}_{S}\right) \stackrel{\Delta \mathrm{r}_{S}}{\longrightarrow} \Delta S\left(\mathbb{X}_{I d}\right) .
$$

Then $\mathrm{r}_{\Delta S}(x)$ is the measure on $S\left(\mathbb{X}_{I d}\right)$ having

$$
\mathrm{r}_{\Delta S}(x)(A)=\mu_{x}\left(\mathrm{r}_{S}^{-1}(A)\right)
$$

for all measurable subsets $A$ of $S\left(\mathbb{X}_{I d}\right)$.

The derivation of $(5.9)$ in this case follows the pattern of [MV06, p. 626]:

$$
\begin{array}{rlrl}
\mathrm{r}_{\Delta S}^{-1}\|[\geqslant p] \varphi\|_{\Delta S} & =\rho_{\Delta S}^{-1}\left(\left(\Delta \mathrm{r}_{S}\right)^{-1}\left(\beta^{p}\|\varphi\|_{S}\right)\right) & \\
& =\rho_{\Delta S}^{-1}\left(\beta^{p}\left(\mathrm{r}_{S}^{-1}\|\varphi\|_{S}\right)\right) & & \text { by }(2.1) \\
& =\rho_{\Delta S}^{-1}\left(\beta^{p}\left(|\varphi|_{S}\right)\right) & & \text { hypothesis on } \mathrm{r}_{S} \\
& =|[\geqslant p] \varphi|_{\Delta S} &
\end{array}
$$

Definition 5.8 (The Canonical T-Coalgebra for $D$ ) For a Lindenbaum T-deduction system $D$, the canonical $T$-coalgebra for $D$ is $\left(\mathbb{X}^{D}, \alpha^{D}\right)$, where $\mathbb{X}^{D}$ is $\mathbb{X}_{I d}$, and $\alpha^{D}$ is $\boldsymbol{r}_{T} \circ \rho_{I d}$ :

$$
\mathbb{X}_{I d} \stackrel{\rho_{I d}}{\longrightarrow} \mathbb{X}_{T} \stackrel{\mathrm{r}_{T}}{\longrightarrow} T\left(\mathbb{X}_{I d}\right) .
$$

Lemma 5.9 (The Truth Lemma) For any $\varphi: S$,

(1) $\llbracket \varphi \rrbracket_{S}^{\alpha^{D}}=\|\varphi\|_{S}$.

(2) For all $x \in \mathbb{X}_{S}, \quad \alpha^{D}, \mathrm{r}_{S}(x) \models_{S} \varphi \quad$ iff $\varphi \in x$.

Proof.

(1) By induction on $\varphi$. We have $\llbracket \perp_{S} \rrbracket_{S}^{\alpha^{D}}=\emptyset=\left\|\perp_{S}\right\|_{S}$ and $\llbracket A \rrbracket_{\mathbb{X}}^{\alpha^{D}}=A=\|A\|_{\mathbb{X}}$ by definition. In fact the match between the definitions of $\llbracket \varphi \rrbracket_{S}^{\alpha}$ and $\|\varphi\|_{S}$ make all the inductive cases routine except for $[$ next $] \varphi: I d$, where $\varphi: T$. But

$$
\begin{array}{rlrl}
\llbracket \text { next } \varphi \rrbracket_{I d}^{\alpha^{D}} & =\left(\alpha^{D}\right)^{-1} \llbracket \varphi \rrbracket_{T}^{\alpha^{D}} & & \text { by definition } \\
& =\left(\alpha^{D}\right)^{-1}\|\varphi\|_{T} & & \text { by induction hypothesis } \\
& =\rho_{I d}^{-1}\left(\mathrm{r}_{T}^{-1}\|\varphi\|_{T}\right) & \\
& =\rho_{I d}^{-1}|\varphi|_{T} & & \text { by Lemma } 5.7 \\
& =\mid\left.[\text { next }] \varphi\right|_{I d} & & \text { by }(5.7) \\
& =\|[\text { next }] \varphi \|_{I d} . &
\end{array}
$$


(2) From (1) and Lemma 5.7, $\mathrm{r}_{S}^{-1} \llbracket \varphi \rrbracket_{S}^{\alpha^{D}}=|\varphi|_{S}$, which is what (2) says.

Corollary 5.10 des $_{S}^{\alpha^{D}} \circ \mathrm{r}_{S}$ is the identity function on $X_{S}$. Hence if $D$ is a sound deduction system, then $X_{S}$ is the set of all $S$-description sets.

Proof. For each $x \in X_{S}$, by definition

$$
\operatorname{des}_{S}^{\alpha^{D}}\left(\mathrm{r}_{S}(x)\right)=\left\{\varphi: S \mid \alpha^{D}, \mathrm{r}_{S}(x) \models_{S} \varphi\right\} .
$$

But by (2) of the Truth Lemma, this is just $\{\varphi \mid \varphi \in x\}=x$.

Hence each $\vdash_{S}$-maximal $x$ is the description set $\operatorname{des}_{S}^{\alpha^{D}}\left(r_{S}(x)\right)$. But if $D$ is sound, every $S$-description set is $\vdash_{S}$-maximal by Theorem $4.17(2)$.

In [Vig05, MV06] a final $T$-coalgebra is constructed from spaces $S^{*}$ based directly on the set of all $S$-description sets. We have arrived at a proof-theoretic construction of the same spaces from a sound Lindenbaum $T$-deduction system. We will shortly show (in Corollary 5.15) that this system must be Conseq $_{T}$. But first we confirm that connection between $\mathrm{r}_{S}$ and $d e s_{S}^{\alpha^{D}}$ is even tighter than indicated above:

Theorem 5.11 $\mathrm{r}_{S}$ and des $_{S}^{\alpha^{D}}$ are mutually inverse and give an isomorphism between the measurable spaces $\mathbb{X}_{S}$ and $S\left(\mathbb{X}_{I d}\right)=S\left(\mathbb{X}^{D}\right)$.

Proof. Let $|\varphi|_{S} \in \mathcal{A}_{S}^{D}$ be a generating measurable set from $\mathbb{X}_{S}$ (Definition 5.1). Then

$$
\left(d e s_{S}^{\alpha^{D}}\right)^{-1}|\varphi|_{S}=\left\{y \mid \varphi \in \operatorname{des}_{S}^{\alpha^{D}}(y)\right\}=\llbracket \varphi \rrbracket_{S}^{\alpha^{D}},
$$

which is a measurable subset of $S\left(\mathbb{X}_{I d}\right)$ as $\varphi:: S$. Hence $\operatorname{des}_{S}^{\alpha^{D}}: S\left(\mathbb{X}_{I d}\right) \rightarrow \mathbb{X}_{S}$ is measurable.

It remains to show that $\mathrm{r}_{S} \circ \operatorname{des}_{S}^{\alpha^{D}}$ is the identity function. This requires a consideration of cases, by induction on $S$.

- $S=I d$. Here $\mathbf{r}_{I d}$ is the identity function on $X_{I d}$. Hence by Corollary $5.10, d_{e} s_{I d}^{\alpha^{D}}$ is also the identity function on $X_{I d}$.

- $S=\mathbb{X}$. Then for $x \in X_{\mathbb{X}}, r_{\mathbb{X}}(x)=$ the unique $c \in X$ such that $\{c\} \in x$. Thus if $c$ belongs to $\mathbb{X}$, and $x=\operatorname{des}_{\mathbb{X}}^{\alpha^{D}}(c)$, then $\{c\} \in x$ as $\alpha^{D}, c \models_{\mathbb{X}}\{c\}$, so $\boldsymbol{r}_{X}\left(d e s_{\mathbb{X}}^{\alpha^{D}}(c)\right)=r_{\mathbb{X}}(x)=c$ as required.

- $S=S_{1} \times S_{2}$ : assume the result for $S_{1}$ and $S_{2}$. Take $z=\left(z_{1}, z_{2}\right)$ in $S_{1}\left(\mathbb{X}_{I d}\right) \times S_{2}\left(\mathbb{X}_{I d}\right)$, and let $x=\operatorname{des}_{S_{1} \times S_{2}}^{\alpha^{D}}(z)$. We want $\mathrm{r}_{S_{1} \times S_{2}}(x)=z$.

Now for $\varphi: S_{j}, \varphi \in\left[\mathrm{pr}_{j}\right]^{-} x$ iff $\alpha^{D}, \pi_{j}(z) \models S_{j} \varphi$, hence $\left[\mathrm{pr}_{j}\right]^{-} x=\operatorname{des}_{S_{j}}^{\alpha^{D}}\left(z_{j}\right)$. Thus $\mathrm{r}_{S_{j}}\left(\left[\mathrm{pr}_{j}\right]^{-} x\right)=\mathrm{r}_{S_{j}}\left(\operatorname{des}_{S_{j}}^{\alpha^{D}}\left(z_{j}\right)\right)=z_{j}$ by hypothesis on $S_{j}$. It follows from (5.10) that $\mathrm{r}_{S_{1} \times S_{2}}(x)=\left(z_{1}, z_{2}\right)$ as required.

- $S=S_{1}+S_{2}$ : Take $z$ in $S_{1}\left(\mathbb{X}_{I d}\right)+S_{2}\left(\mathbb{X}_{I d}\right)$, with $z=i n_{j}\left(z_{j}\right)$ for some $j$ and some $z_{j}$ in $S_{j}\left(\mathbb{X}_{I d}\right)$. Let $x=\operatorname{des}_{S_{1}+S_{2}}^{\alpha^{D}}(z)$. Then $\neg\left[\mathrm{in}_{j}\right] \perp_{S_{j}} \in x$, so $\mathrm{r}_{S_{1}+S_{2}}(x)=i n_{j}\left(\mathrm{r}_{S_{j}}\left(\left[\mathrm{in}_{j}\right]^{-} x\right)\right)$ by (5.11).

Now for $\varphi: S_{j}, \varphi \in\left[\mathrm{in}_{j}\right]^{-} x$ iff $\alpha^{D}, z_{j} \models_{S_{j}} \varphi$, hence $\left[\mathrm{in}_{j}\right]^{-} x=\operatorname{des}_{S_{j}}^{\alpha^{D}}\left(z_{j}\right)$. Thus $\mathrm{r}_{S_{j}}\left(\left[\mathrm{in}_{j}\right]^{-} x\right)=$ $\mathrm{r}_{S_{j}}\left(\operatorname{des}_{S_{j}}^{\alpha^{D}}\left(z_{j}\right)\right)=z_{j}$ by hypothesis on $S_{j}$. Hence $\mathrm{r}_{S_{1}+S_{2}}(x)=i n_{j}\left(z_{j}\right)=z$ as required. 
- If $S^{E} \in \operatorname{lng} T$, assume the result for $S$. Take $f \in\left(S\left(\mathbb{X}_{I d}\right)\right)^{E}$, and let $x=\operatorname{des}_{S^{E}}^{\alpha^{D}}(f)$. We want $\mathrm{r}_{S E}(x)=f$.

Now for $\varphi: S$ and $e \in E, \varphi \in\left[\mathrm{ev}_{e}\right]^{-} x$ iff $\alpha^{D}, f(e) \models_{S} \varphi$, hence $\left[\operatorname{ev}_{e}\right]^{-} x=\operatorname{des}_{S}^{\alpha^{D}}(f(e))$. Thus $\mathrm{r}_{S}\left(\left[\operatorname{ev}_{e}\right]^{-} x\right)=\mathrm{r}_{S}\left(\operatorname{des}_{S}^{\alpha^{D}}(f(e))\right)=f(e)$ by hypothesis on $S$. Since this holds for all $e \in E$, it follows from (5.12) that $\mathrm{r}_{S^{E}}(x)=f$ as required.

- If $\Delta S \in \operatorname{lng} T$, assume the result for $S$. Take a member $\mu$ of $\Delta S\left(\mathbb{X}_{I d}\right)$ and let $x=\operatorname{des}_{\Delta S}^{\alpha^{D}}(\mu)$. Now by the induction hypothesis, $r_{S}$ is an isomorphism between $\mathbb{X}_{S}$ and $S\left(\mathbb{X}_{I d}\right)$, so as $\mathbb{X}_{S}$ is generated by the algebra of sets $|\varphi|_{S}$ with $\varphi:: S, S\left(\mathbb{X}_{I d}\right)$ is generated by their $\mathrm{r}_{S}$-images $\mathrm{r}_{S}|\varphi|_{S}$. Hence by (5.13), the measure $\mathrm{r}_{\Delta S}(x)$ has

$$
\mathrm{r}_{\Delta S}(x)\left(\mathrm{r}_{S}|\varphi|_{S}\right)=\mu_{x}\left(\left(\mathrm{r}_{S}\right)^{-1} \mathrm{r}_{S}|\varphi|_{S}\right)=\mu_{x}\left(|\varphi|_{S}\right) .
$$

By the Truth Lemma, $|\varphi|_{S}=\mathrm{r}_{S}^{-1} \llbracket \varphi \rrbracket_{S}^{\alpha^{D}}$, so $r_{S}|\varphi|_{S}=\llbracket \varphi \rrbracket_{S}^{\alpha^{D}}$, hence $\mu\left(\mathrm{r}_{S}|\varphi|_{S}\right) \geqslant q$ iff $\mu\left(\llbracket \varphi \rrbracket_{S}^{\alpha^{D}}\right) \geqslant q$ iff $[\geqslant q] \varphi \in \operatorname{des}_{\Delta S}^{\alpha^{D}}(\mu)=x$. Thus

$$
\mu\left(\mathrm{r}_{S}|\varphi|_{S}\right)=\sup \left\{q \in[0,1]_{\mathbb{Q}} \mid[\geqslant q] \varphi \in x\right\}=\mu_{x}\left(|\varphi|_{S}\right) .
$$

Since any probability measure is determined by its values on a generating algebra, this shows that $\mathrm{r}_{\Delta S}(x)=\mu$ as required.

We are now ready to give a characterisation of the semantic consequence relations $\models_{S}$.

Definition 5.12 A T-deduction system $\left\{\vdash_{S} \mid S \in \operatorname{lng} T\right\}$ is called complete if, for all $S$ and all $\Gamma \cup\{\varphi\} \subseteq$ Form $_{S}, \Gamma \models_{S} \varphi$ implies $\Gamma \vdash_{S} \varphi$.

With the help of Lemma 4.5(5), one can show

Lemma 5.13 A T-deduction system is complete iff every $\vdash_{S}$-consistent set of formulas is satisfiable in some T-coalgebra.

Theorem 5.14 (Completeness) For any Lindenbaum $T$-deduction system $D=\left\{\vdash_{S}^{D} \mid S \in\right.$ $\operatorname{lng} T\}$,

(1) $\Gamma \models_{S}^{\alpha^{D}} \varphi$ if, and only if, $\Gamma \vdash_{S}^{D} \varphi$.

(2) $\Gamma \models_{S} \varphi$ implies $\Gamma \vdash_{S}^{D} \varphi$.

Proof. For (1), suppose first that $\Gamma \nvdash_{S}^{D} \varphi$. Then $\Gamma \cup\{\neg \varphi\}$ is $\vdash_{S}^{D}$-consistent by Lemma 4.5(5). Then the Lindenbaum property implies that $\Gamma \cup\{\neg \varphi\} \subseteq x$ for some $x \in X_{S}^{D}$. Hence by the Truth Lemma $5.9(2), \alpha^{D}, \mathrm{r}_{S}(x) \models \Gamma$ and $\alpha^{D}, \mathrm{r}_{S}(x) \not \models \varphi$, so $\Gamma \not \neq_{S}^{\alpha^{D}} \varphi$.

Conversely, if $\Gamma \forall_{S}^{\alpha^{D}} \varphi$, then for some $y \in S\left(\mathbb{X}_{I d}\right), \alpha^{D}, y \models \Gamma$ and $\alpha^{D}, y \not \models \varphi$. But by Theorem 5.11, $y=\mathrm{r}_{S}(x)$ for some $x \in X_{S}^{D}$, and hence by the Truth Lemma, $\Gamma \subseteq x$ and $\varphi \notin x$. $\vdash{ }_{S}^{D}$-closure of $x$ (Corollary 4.14) then ensures that $\Gamma \nvdash_{S}^{D} \varphi$.

(2) follows from (1), since $\Gamma \models_{S} \varphi$ implies $\Gamma \models_{S}^{\alpha^{D}} \varphi$ by definition of $\models_{S}$.

Corollary 5.15 If $D$ is any sound Lindenbaum $T$-deduction system, then in general

$$
\Gamma \models_{S} \varphi \quad \text { iff } \quad \Gamma \models_{S}^{\alpha^{D}} \varphi \quad \text { iff } \quad \Gamma \vdash_{S}^{D} \varphi .
$$

Consequently, Conseq ${ }_{T}=\left\{\models_{S} \mid S \in \operatorname{lng} T\right\}$ is the unique sound Lindenbaum T-deduction system. 
Another way to express this is that there is no Lindenbaum deductive system that is a proper subsystem of Conseq $q_{T}$. In making such comparisons between Ing $T$-indexed collections, we partially order them by pointwise inclusion, writing

$$
\left\{Y_{S} \mid S \in \operatorname{lng} T\right\} \leq\left\{Z_{S} \mid S \in \operatorname{lng} T\right\}
$$

to mean that $Y_{S} \subseteq Z_{S}$ for all $S$. Soundness of $D$ then just means that $D \leq$ Conseq $_{T}$, while completeness means Conseq $_{T} \leq D$.

An Ing $T$-indexed collection is the least one having a certain property if it is least in the sense of this ordering $\leq$. Typically we deal with properties that are preserved by pointwise intersection. In other words, if we are given, for each $i \in I$, a collection $\left\{Y_{S}^{i} \mid S \in \operatorname{lng} T\right\}$ satisfying the property, then $\left\{\bigcap_{i \in I} Y_{S}^{i} \mid S \in \operatorname{lng} T\right\}$ will also satisfy the property. This implies that there is a $\leq$-least collection/system with the property, and indeed a least one that $\leq$ extends any specified system. For example, there is a least T-deduction system, which we denote $D_{T}^{\lambda}=\left\{\vdash_{S}^{\lambda} \mid S \in \operatorname{lng} T\right\}$.

It could be said that the characterisation of Conseq $_{T}$ in Corollary 5.15 is not purely prooftheoretic, since it involves the semantic criterion of soundness, or reference to Conseq $_{T}$ itself. In response, we could focus on $D_{T}^{\lambda}$, which is sound because $D_{T}^{\lambda} \leq$ Conseq $_{T}$ by definition of "least". Then we have that

$$
D_{T}^{\lambda} \text { is complete iff } D_{T}^{\lambda}=\text { Conseq }_{T} \quad \text { iff } \quad D_{T}^{\lambda} \text { is Lindenbaum. }
$$

But it turns out that there are functors $T$ for which $D_{T}^{\lambda}$ is not Lindenbaum, even polynomial ones not involving $\Delta$. An example of such a functor over Set is given in [FG06], and this adapts readily to one over Meas, as follows.

Let $N$ be the constant functor given by the space $\omega$ of natural numbers with just $\omega$ and $\emptyset$

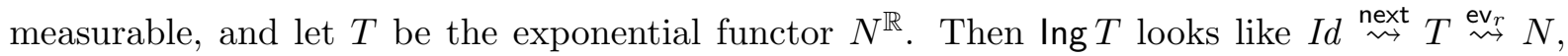
with an edge $\stackrel{\mathrm{ev}_{r}}{\rightsquigarrow}$ for each real $r \in \mathbb{R}$. For each $r \in \mathbb{R}$ and $n \in \omega$, let $(r \mapsto n)$ be the formula $\left[\right.$ next] $\left[\mathrm{ev}_{r}\right]\{n\}$ of sort $I d$. A $T$-coalgebra $(\mathbb{X}, \alpha)$ has a transition function $\alpha: X \rightarrow \omega^{\mathbb{R}}$, with $\alpha, x=_{I d}(r \mapsto n)$ iff $\alpha(x)(r)=n$. Let

$$
\Gamma_{\mathbb{R}}=\{(r \mapsto n) \rightarrow \neg(s \mapsto n) \mid r, s \in \mathbb{R}, r \neq s, n \in \omega\} .
$$

Then $\Gamma_{\mathbb{R}}$ is unsatisfiable, and hence is not included in any description set. For, if we had $\alpha, x \models_{I d} \Gamma_{\mathbb{R}}$, then the function $f: \mathbb{R} \rightarrow \omega$ defined by $f(r)=\alpha(x)(r)$ would be injective in view of the satisfaction of the members of $\Gamma_{\mathbb{R}}$. But of course no such injection exists.

Now in [FG06] a one-sorted deduction system for $T$ was defined for which $\Gamma_{\mathbb{R}}$ is consistent. This adapts to an $\operatorname{lng}_{T}$-sorted deduction system for which $\Gamma_{\mathbb{R}}$ is $\vdash_{I d}$-consistent (see Section 8). Consequently $\Gamma_{\mathbb{R}}$ is $\vdash_{I d^{\lambda}}^{\lambda}$-consistent.

Hence if $D_{T}^{\lambda}$ were Lindenbaum, then $\Gamma_{\mathbb{R}}$ would be included in some $\vdash_{I d^{-}}^{\lambda}$-maximal set $x$, and so would be satisfiable at $x$ in the canonical $T$-coalgebra for $D_{T}^{\lambda}$ - which we just saw is impossible. So this $D_{T}^{\lambda}$ is not Lindenbaum, is not equal to Conseq $_{T}$, and is not complete: $\Gamma_{\mathbb{R}} \models_{I d} \perp$ but $\Gamma_{\mathbb{R}} \nvdash_{I d}^{\lambda} \perp$.

Finally then, to obtain a genuinely proof-theoretic characterisation of Conseq $_{T}$, we consider the preservation of the Lindenbaum property under pointwise intersection:

Lemma 5.16 If, for each $i \in I, D^{i}=\left\{\vdash_{S}^{i} \mid S \in \operatorname{Ing} T\right\}$ is a Lindenbaum T-deduction system, and $D=\left\{\vdash_{S}^{D} \mid S \in \operatorname{Ing} T\right\}$ is the pointwise intersection of $\left\{D^{i} \mid i \in I\right\}$, then $D$ is Lindenbaum.

Proof. If $\Gamma$ is $\vdash_{S}^{D}$-consistent, then $\Gamma \nvdash_{S}^{D} \perp_{S}$, so for some $i \in I, \Gamma \nvdash_{S}^{i} \perp_{S}$. But $D^{i}$ is Lindenbaum, so there is some negation-complete $\vdash_{S}^{i}$-consistent set $\Gamma^{\prime}: S$ extending $\Gamma$. But as $\vdash_{S}^{D}$ is included in $\vdash_{S}^{i}, \Gamma^{\prime}$ is also $\vdash_{S}^{D}$-consistent, and hence is $\vdash_{S}^{D}$-maximal.

In particular, there exists a least Lindenbaum T-deduction system, the intersection of all Lindenbaum systems. But this is just Conseq $_{T}$. To sum up: 
Theorem 5.17 For any measurable polynomial functor $T$,

(1) The local semantic consequence systems Conseq $_{T}^{\alpha}=\left\{\models_{S}^{\alpha} \mid S \in \operatorname{lng} T\right\}$ are exactly the Lindenbaum T-deduction systems.

(2) The global consequence system Conseq ${ }_{T}=\left\{\models_{S} \mid S \in \operatorname{Ing} T\right\}$ over the class of all T-coalgebras is the least Lindenbaum T-deduction system.

Proof. (1): Each Conseq ${ }_{T}^{\alpha}$ is Lindenbaum by Theorem 4.17(3). Conversely, if $D$ is Lindenbaum, then Theorem 5.14(1) states that $D=$ Conseq $_{T}^{\alpha_{D}}$.

(2): Conseq $_{T}$ is the intersection of all the semantic consequence systems Conseq ${ }_{T}^{\alpha}$, hence by (1) is the intersection of all Lindenbaum systems.

Alternatively, note that Conseq $_{T}$ is Lindenbaum by Theorem 4.17(4), while by the Completeness Theorem 5.14(2), Conseq $_{T} \leq D$ whenever $D$ is Lindenbaum.

\section{A Countable Language for $[0,1]$}

If $T$ has an uncountable space $\mathbb{X}$ is an ingredient, then there are uncountably many formulas of sort $\mathbb{X}$, including all the singletons of $\mathbb{X}$. But one might suspect that some separable uncountable spaces can be handled by a countable syntax. We now show how this can be done for the unit interval $[0,1]$.

Let $\mathbb{I}^{g}$ be the countable set of all subintervals $[p, q]$ of $[0,1]$ that have rational end-points $p \leq q$, and let $\mathbb{I}$ be the space $\left([0,1], \sigma\left(\mathbb{I}^{g}\right)\right)$. The measurable sets of $\mathbb{I}$ are the Borel sets for the usual topology on $[0,1]$. If $\mathbb{I}$ is an ingredient of some measurable polynomial functor $T$, then as formulas of sort $\mathbb{I}$ we take just the members $[p, q]$ of $\mathbb{I}^{g}$. The semantics of formulas is as usual, so

$$
\alpha, x \models_{\mathbb{I}}[p, q] \quad \text { iff } \quad p \leqslant x \leqslant q .
$$

We still have some singleton formulas, since $\{p\}=[p, p]$, but not enough to formulate the Constant Rule for $\vdash_{\mathbb{I}}$ of Definition 4.4. Instead we take the rules

$\left(\mathbb{I}_{p}^{-}\right) \quad\{[q, 1] \mid q<p\} \vdash_{\mathbb{I}}[p, 1]$

$\left(\mathbb{I}_{p}^{+}\right) \quad\{[0, q] \mid p<q\} \vdash_{\mathbb{I}}[0, p]$

for all $p \in[0,1]_{\mathbb{Q}}$. In place of the group 2 axioms of Definition 4.1, we take the following for $A x_{\mathbb{I}}$ :

(II.1) $[q, 1] \rightarrow[p, 1] \wedge \neg[0, p] \quad$ if $p<q$

(II.2) $\neg[p, 1] \rightarrow[0, q] \quad$ if $p<q$

$$
[p, 1] \wedge[0, q] \leftrightarrow[p, q] \quad \text { if } p \leqslant q
$$

In carrying out the analysis of the previous Section, the only new consideration arises in Lemma 5.7, where we must now define a measurable function $r_{\mathbb{I}}: \mathbb{X}_{\mathbb{I}} \rightarrow \mathbb{I}$ such that for all formulas $\varphi: \mathbb{I}$ we get $\mathbf{r}_{\mathbb{I}}^{-1}\|\varphi\|_{\mathbb{I}}=|\varphi|_{\mathbb{I}}$. For this we put

$$
\mathbf{r}_{\mathbb{I}}(x)=\sup \left\{q \in[0,1]_{\mathbb{Q}} \mid[q, 1] \in x\right\} .
$$

Letting $p=0$ in rule $\left(\mathbb{I}_{p}^{-}\right)$shows $\vdash_{\mathbb{I}}[0,1]$, hence for any $x \in X_{\mathbb{I}}$ we have $[0,1] \in x$ and therefore $\mathbf{r}_{\mathbb{I}}(x)$ is defined.

\section{Lemma 6.1}


(1) $\boldsymbol{r}_{\mathbb{I}}(x) \geqslant p$ iff $[p, 1] \in x$.

(2) $\boldsymbol{r}_{\mathbb{I}}(x) \leqslant p$ iff $[0, p] \in x$.

Proof.

(1) From right to left holds by definition of $\mathbf{r}_{\mathbb{I}}$. For the converse: if $\mathbf{r}_{\mathbb{I}}(x) \geqslant p$, then for each $q<p$ there must be some rational $r>q$ with $[r, 1] \in x$, hence $[q, 1] \in x$ using $\vdash_{\mathbb{I}}[r, 1] \rightarrow[q, 1]$ by axiom (II.1). Therefore $[p, 1] \in x$ by rule $\left(\mathbb{I}_{p}^{-}\right)$and the $\vdash_{\mathbb{I}}$-closure of $x$.

(2) Let $[0, p] \in x$. If we had $\mathbf{r}_{\mathbb{I}}(x) \nless p$, we could take a rational $q$ with $p<q \leqslant \mathbf{r}_{\mathbb{I}}(x)$ and get $[q, 1] \in x$ by part (1). But $\neg[0, p] \notin x$, from which by axiom (II.1) and properties of maximal sets we get the contradictory $[q, 1] \notin x$. Hence $\mathbf{r}_{\mathbb{I}}(x) \leqslant p$.

Conversely, suppose $\mathbf{r}_{\mathbb{I}}(x) \leqslant p$. To show $[0, p] \in x$, it is enough by rule $\left(\mathbb{I}_{p}^{+}\right)$to show that $[0, q] \in x$ for all $p<q$. But if $p<q$, take a rational $r$ with $p<r<q$. Since $\boldsymbol{r}_{I}(x) \ngtr r$, part $(1)$ gives $[r, 1] \notin x$, so $\neg[r, 1] \in x$. Since $\vdash_{\mathbb{I}} \neg[r, 1] \rightarrow[0, q]$ by axiom (I.2), this yields $[0, q] \in x$ as desired.

Corollary $6.2 \mathrm{r}_{\mathbb{I}}(x) \in[p, q]$ iff $[p, q] \in x$.

Proof. Using the Lemma, $\mathbf{r}_{\mathbb{I}}(x) \in[p, q]$ iff $[p, 1],[0, q] \in x$, which is equivalent to $[p, 1] \wedge[0, q] \in x$, which in turn is equivalent to $[p, q] \in x$ by axiom (I.3).

Since $\|[p, q]\|_{\mathbb{I}}=[p, q]$, this Corollary ensures that $\mathbf{r}_{\mathbb{I}}^{-1}\|A\|_{\mathbb{I}}=|A|_{\mathbb{I}}$ for all $A \in \mathbb{I}^{g}$, which is enough to guarantee the measurability of $r_{\mathbb{I}}$ and complete Lemma 5.7 for this case.

\section{Kripke Polynomial Functors}

A polynomial functor $T$ : Set $\rightarrow$ Set is any functor that can be constructed in finitely many steps from constant functors and/or the identity functor by forming products, coproducts and exponentials. A Kripke polynomial functor (KPF) is one whose formation permits also composition $\mathcal{P} T=\mathcal{P} \circ T$ with the covariant powerset functor $\mathcal{P}$. The papers [Röß00, Jac01] give deduction systems for the logic of coalgebras for KPF's whose constant ingredients $\mathbb{X}$ have a finite set $X$ as constant value. The methodology of this paper adapts to KPF's with infinite constant sets, as follows.

The syntax for products, coproducts and exponentials remains the same. In the definition of $\operatorname{lng} T, \Delta$ is replaced by $\mathcal{P}$. Instead of the probabilistic constructors $\geqslant p$ we have the single constructor $\mathcal{P}$, with edges in $\operatorname{lng} T$ of the form $\mathcal{P} S \stackrel{\mathcal{P}}{\rightsquigarrow} S$, whereby for each formula $\varphi: S$ there is a formula $[\mathcal{P}] \varphi: \mathcal{P} S$. This has semantics

$$
\llbracket[\mathcal{P}] \varphi \rrbracket_{\mathcal{P} S}^{\alpha}=\mathcal{P}\left(\llbracket \varphi \rrbracket_{S}^{\alpha}\right),
$$

or equivalently

$$
\alpha, x \models_{\mathcal{P} S}[\mathcal{P}] \varphi \quad \text { iff } \quad y \in x \text { implies } \alpha, y \models_{S} \varphi .
$$

In the definition of axioms and $T$-deduction systems, the group 7 axioms for the probabilistic modalities are deleted along with the the Archimedean and Countable Additivity Rules, and the Definite Box Rule just becomes the Box Rule stated for every edge $\stackrel{\kappa}{\rightsquigarrow}$. Then as in Lemma 4.6(4) we can derive

$$
\vdash_{\mathcal{P} S}[\mathcal{P}](\varphi \rightarrow \psi) \rightarrow([\mathcal{P}] \varphi \rightarrow[\mathcal{P}] \psi)
$$


In fact no axioms of sort $\mathcal{P} S$ are needed.

In working with canonical sets $X_{S}^{D}$ for a deductive system $D$ for a Lindenbaum functor $T$, we use the functions $\rho_{S}$ as given in Lemma 5.6, except that there is no longer a reference to measurability, and in place of $\rho_{\Delta S}$ we require a function

$$
X_{\mathcal{P} S} \stackrel{\rho_{\mathcal{P} S}}{\longrightarrow} \mathcal{P}\left(X_{S}\right)
$$

satisfying

$$
\rho_{\mathcal{P} S}^{-1}\left(\mathcal{P}|\varphi|_{S}\right)=|[\mathcal{P}] \varphi|_{\mathcal{P} S}
$$

For this we define, as in classical modal logic,

$$
\rho_{\mathcal{P} S}(x)=\left\{y \in X_{S} \mid[\mathcal{P}]^{-} x \subseteq y\right\},
$$

where $[\mathcal{P}]^{-} x=\{\varphi: S \mid[\mathcal{P}] \varphi \in x\}$ as usual. Equivalently,

$$
\rho_{\mathcal{P} S}(x)=\bigcap\left\{|\varphi|_{S} \mid[\mathcal{P}] \varphi \in x\right\},
$$

from which it is immediate that $x \in|[\mathcal{P}] \varphi|_{\mathcal{P} S}$ implies $\rho_{\mathcal{P} S}(x) \subseteq|\varphi|_{S}$, as required for one direction of (7.2). Conversely, if $\rho_{\mathcal{P} S}(x) \subseteq|\varphi|_{S}$, then $[\mathcal{P}]^{-} x \vdash_{S} \varphi$, or else $[\mathcal{P}]^{-} x \cup\{\neg \varphi\}$ would be $\vdash_{S^{-}}$ consistent, hence extendable by the Lindenbaum property to a $y \in \rho_{\mathcal{P} S}(x)-|\varphi|_{S}$. But from $[\mathcal{P}]^{-} x \vdash_{S} \varphi$ we obtain $\{[\mathcal{P}] \psi \mid[\mathcal{P}] \psi \in x\} \vdash_{\mathcal{P} S}[\mathcal{P}] \varphi$ by the Box Rule, hence $x \vdash_{\mathcal{P} S}[\mathcal{P}] \varphi$ by Monotonicity (Lemma 4.5), then $[\mathcal{P}] \varphi \in x$ by $\vdash_{S}$-closure of $x$ (Corollary 4.14). This gives $x \in|[\mathcal{P}] \varphi|_{\mathcal{P} S}$ to complete the proof of (7.2).

Lemma 5.7 now becomes the result that there are functions $\mathrm{r}_{S}: X_{S} \rightarrow S\left(X_{I d}\right)$ with $\mathrm{r}_{S}^{-1}\|\varphi\|_{S}=|\varphi|_{S}$. The new case of $\mathcal{P} S \in \operatorname{lng} T$ requires us to define

$$
\|[\mathcal{P}] \varphi\|_{\mathcal{P} S}=\mathcal{P}\|\varphi\|_{S} \subseteq \mathcal{P} S\left(X_{I d}\right),
$$

and put $\mathrm{r}_{\mathcal{P} S}=\mathcal{P r}_{S} \circ \rho_{\mathcal{P} S}$ :

$$
X_{\mathcal{P} S} \stackrel{\rho_{\mathcal{P} S}}{\longrightarrow} \mathcal{P}\left(X_{S}\right) \stackrel{\mathcal{P r}_{S}}{\longrightarrow} \mathcal{P} S\left(X_{I d}\right),
$$

where $\mathcal{P r}_{S}(Y)$ is the $\mathrm{r}_{S}$-image $\mathrm{r}_{S}[Y]$ of any $Y \subseteq X_{S}$.

Now assuming that $\mathrm{r}_{S}^{-1}\|\varphi\|_{S}=|\varphi|_{S}$, then for each $Y \subseteq X_{S}$,

$$
\mathrm{r}_{S}[Y] \subseteq\|\varphi\|_{S} \text { iff } Y \subseteq \mathrm{r}_{S}^{-1}\|\varphi\|_{S}=|\varphi|_{S},
$$

so $\left(\mathcal{P r}_{S}\right)^{-1}\left(\mathcal{P}\|\varphi\|_{S}\right)=\left\{Y \subseteq X_{S} \mid \mathrm{r}_{S}[Y] \subseteq\|\varphi\|_{S}\right\}=\mathcal{P}|\varphi|_{S}$, hence

$$
\mathrm{r}_{\mathcal{P} S}^{-1}\|[\mathcal{P}] \varphi\|_{\mathcal{P} S}=\rho_{\mathcal{P} S}^{-1}\left(\left(\mathcal{P r}_{S}\right)^{-1}\left(\mathcal{P}\|\varphi\|_{S}\right)\right)=\rho_{\mathcal{P} S}^{-1}\left(\mathcal{P}|\varphi|_{S}\right),
$$

from which by $(7.2)$ we get $\mathbf{r}_{\mathcal{P} S}^{-1}\|[\mathcal{P}] \varphi\|_{\mathcal{P} S}=|[\mathcal{P}] \varphi|_{\mathcal{P} S}$ as desired.

The rest of the theory then goes through unchanged, to construct a canonical $T$-coalgebra $\left(X^{D}, \alpha^{D}\right)$ for any deduction system $D$ for a Lindenbaum KPF $T$. This satisfies the Truth Lemma 5.9, and leads to a proof that $D$ is complete, and hence that Conseq $_{T}$ is the only sound Lindenbaum $T$-deduction system.

\section{A Specific Deduction System}

For each Kripke polynomial functor $T$ a particular deduction system $D_{T}^{+}=\left\{\vdash_{S}^{+} \mid S \in \operatorname{lng} T\right\}$ can be defined that is sound, and is also Lindenbaum when the set of all formulas is countable. In the latter case, $D_{T}^{+}$must be identical to $\operatorname{Conseq}_{T}$. For polynomial functors, this theory was worked out in [FG06] using a one-sorted language (essentially the $I d$-formulas). Here we describe a many-sorted version for KPF's. 
Working with an infinitary Hilbert-style proof system would require the use of transfinite proof-sequences or infinitely-branching proof trees. We prefer instead a more basic set-theoretic approach that comes from the general theory of inductive definitions [Acz77].

The essential idea is to introduce a new notion of an $\mathcal{R}_{S}$-theory, a set of formulas closed under axioms and rules of inference to be specified below. Then the set $\left\{\varphi \mid \Gamma \vdash_{S}^{+} \varphi\right\}$ of formulas that are $\vdash_{S}^{+}$-deducible from $\Gamma$ is defined to be the $\mathcal{R}_{S}$-theory generated by $\Gamma$. The theories are the fixed points of the monotonic operator that takes each set of formulas to its closure under the axioms and inference rules. Then $\left\{\varphi \mid \Gamma \vdash_{S}^{+} \varphi\right\}$ is inductively characterised as the least fixed point/theory extending $\Gamma$. For more discussion of this approach, see [FG06, Section 4].

The first step we take is to modify the definition of $A x_{S}$, to put

$$
[\kappa](\varphi \rightarrow \psi) \rightarrow([\kappa] \varphi \rightarrow[\kappa] \psi) \in A x_{S}
$$

for each edge $S \stackrel{\kappa}{\rightsquigarrow} S^{\prime}$ in $\operatorname{lng} T$ and all $\varphi, \psi: S^{\prime}$. Previously we were able to derive this principle from the Box Rule as in Lemma 4.6(4) and (7.1). But now we need it as an axiom in order to derive the Box Rule for $D_{T}^{+}$(see the proof of Lemma 8.6).

\section{Definition 8.1}

(1) An inference rule, or just rule, is a pair $\langle\Sigma, \varphi\rangle$, where $\Sigma$ is a set of formulas, which may be thought of as a set of premisses, and $\varphi$ is a formula, thought of as a conclusion. If $\Sigma=\{\psi\}$, we may write the rule as $\langle\psi, \varphi\rangle$.

(2) $\Gamma$ is closed under the rule $\langle\Sigma, \varphi\rangle$ if $\Sigma \subseteq \Gamma$ implies $\varphi \in \Gamma$, i.e. if $\Sigma \nsubseteq \Gamma$ or $\varphi \in \Gamma$.

(3) If $\mathcal{R}$ is a set of rules, then $\Gamma$ is $\mathcal{R}$-closed if it is closed under every rule belonging to $\mathcal{R}$.

(4) $\left\{\mathcal{R}_{S} \mid S \in \operatorname{lng}_{T}\right\}$ is the smallest system of relations $\mathcal{R}_{S} \subseteq \mathcal{P}\left(\right.$ Form $\left._{S}\right) \times$ Form $_{S}$ that has the following closure properties:

(a) $\left\langle\{\neg\{c\} \mid c \in X\}, \perp_{\mathbb{X}}\right\rangle \in \mathcal{R}_{\mathbb{X}} \quad$ whenever $\mathbb{X} \in \operatorname{Ing}_{T}$;

(b) $\langle\Gamma, \psi\rangle \in \mathcal{R}_{S^{\prime}}$ implies $\langle[\kappa] \Gamma,[\kappa] \psi\rangle \in \mathcal{R}_{S}$ for each edge $S \stackrel{\kappa}{\rightsquigarrow} S^{\prime} \operatorname{in} \operatorname{lng} T$;

(c) $\langle\Gamma, \psi\rangle \in \mathcal{R}_{S}$ implies $\langle\varphi \rightarrow \Gamma, \varphi \rightarrow \psi\rangle \in \mathcal{R}_{S} \quad$ for every $S \in \operatorname{lng} T$ and $\varphi: S$.

(5) $\left\{A x_{S}^{+} \mid S \in \operatorname{lng}_{T}\right\}$ is the smallest system of sets $A x_{S}^{+} \subseteq$ Form $_{S}$ such that

- $A x_{S} \subseteq A x_{S}^{+}$;

- For each edge $S \stackrel{\kappa}{\rightsquigarrow} S^{\prime}, \varphi \in A x_{S^{\prime}}^{+}$implies $[\kappa] \varphi \in A x_{S}^{+}$.

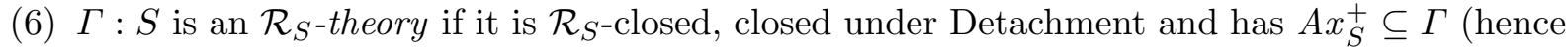
is an $S$-theory).

\section{Theorem 8.2}

(1) If $\varphi \in A x_{S}^{+}$, then $\varphi$ is valid in all $T$-coalgebras.

(2) $\langle\Sigma, \varphi\rangle \in \mathcal{R}_{S}$ implies $\Sigma \models_{S} \varphi$.

Proof.

(1) For any $T$-coalgebra $\alpha$, let $\operatorname{Val}_{S}^{\alpha}=\left\{\varphi \mid \alpha \models_{S} \varphi\right\}$ be the set of all $S$-formulas valid in $\alpha$. Then $A x_{S} \subseteq V_{a l}^{\alpha}$ by Theorem 4.2. Now for each edge $S \stackrel{\kappa}{\rightsquigarrow} S^{\prime}, \alpha=_{S^{\prime}} \varphi$ implies $\alpha=_{S}[\kappa] \varphi$, as is readily checked, so the system $\left\{\operatorname{Val}_{S}^{\alpha} \mid S \in \operatorname{lng} T\right\}$ satisfies the closure conditions of Definition 8.1(5). Since $\left\{A x_{S}^{+} \mid S \in \operatorname{lng} T\right\}$ is defined to be the least such system, $A x_{S}^{+} \subseteq V_{a l}^{\alpha}$ for all $S$ as required. 
(2) The system $\left\{\models_{S} \mid S \in \operatorname{lng} T\right\}$ is a $T$-deduction system (cf. Theorem 4.8), so it satisfies all the closure properties in Definition 8.1(4). Since $\left\{\mathcal{R}_{S} \mid S \in \operatorname{lng}_{T}\right\}$ is the least system with these properties, the result follows.

Corollary 8.3 Every $S$-description set is an $\mathcal{R}_{S}$-theory.

Proof. If $\Gamma=\operatorname{des}_{S}^{\alpha}(x)$ for some state $x$ of a $T$-coalgebra $\alpha$, and $\Sigma \subseteq \Gamma$ with $\langle\Sigma, \varphi\rangle \in \mathcal{R}_{S}$, then $\alpha, x \models_{S} \Sigma$ and $\Sigma \models_{S} \varphi$, so $\alpha, x \models_{S} \varphi$ and hence $\varphi \in \Gamma$. Thus $\Gamma$ is $\mathcal{R}_{S}$-closed. $\Gamma$ includes $A x_{S}^{+}$by Theorem 8.2(1), and is closed under Detachment by the semantics of implication.

Definition 8.4 (The System $D_{T}^{+}$)

Define $\Gamma \vdash_{S}^{+} \varphi$ to mean that $\varphi \in \bigcap\left\{\Sigma \mid \Gamma \subseteq \Sigma\right.$ and $\Sigma$ is an $\mathcal{R}_{S}$-theory $\}$, i.e. $\varphi$ belongs to every $\mathcal{R}_{S}$-theory extending $\Gamma$. In particular, $\vdash_{S}^{+} \varphi$ means that $\varphi$ belongs to every $\mathcal{R}_{S^{-}}$-theory.

It is immediate from this definition and the $\mathcal{R}_{S^{-}}$-closure of $\mathcal{R}_{S^{-}}$-theories that

$$
\langle\Sigma, \varphi\rangle \in \mathcal{R}_{S} \text { implies } \Sigma \vdash_{S}^{+} \varphi .
$$

Note that the intersection of $\mathcal{R}_{S}$-theories is an $\mathcal{R}_{S}$-theory. Hence $\left\{\varphi \mid \Gamma \vdash_{S}^{+} \varphi\right\}$ is the smallest $\mathcal{R}_{S}$-theory including $\Gamma$.

Theorem 8.5 (Soundness) If $\Gamma \vdash_{S}^{+} \varphi$ then $\Gamma \models_{S} \varphi$.

Proof. Suppose $\Gamma \vdash_{S}^{+} \varphi$ and $\alpha, x \models_{S} \Gamma$. We need to show $\alpha, x \models_{S} \varphi$. Since $\operatorname{des}_{S}^{\alpha}(x)$ is an $\mathcal{R}_{S}$-theory by Corollary 8.3, and $\Gamma \subseteq \operatorname{des}_{S}^{\alpha}(x)$, from $\Gamma \vdash_{S}^{+} \varphi$ we get $\varphi \in \operatorname{des}_{S}^{\alpha}(x)$ as required.

We now wish to verify that $D_{T}^{+}=\left\{\vdash_{S}^{+} \mid S \in \operatorname{lng} T\right\}$ is a $T$-deduction system. To begin with, the verification that the relations $\vdash_{S}^{+}$satisfy the Assumption, Modus Ponens, Cut and Deduction Rules of Definition 4.4 proceeds along the lines of [Gol93, Theorem 9.3.3]. In fact the Assumption Rule holds in the stronger form

$$
\varphi \in \Gamma \cup A x_{S}^{+} \text {implies } \Gamma \vdash_{S} \varphi,
$$

because of the inclusion of $A x_{S}^{+}$in every $\mathcal{R}_{S}$-theory.

The implication rules $\langle\varphi \rightarrow \Gamma, \varphi \rightarrow \psi\rangle$ were built into the definition of $\mathcal{R}_{S}$, and hence of $\vdash_{S}^{+}$, because they are needed to establish that $\vdash_{S}^{+}$satisfies the Deduction Rule. Also, all of the results of Lemma 4.5 hold with $\vdash_{S}^{+}$in place of $\vdash_{S}$. Thus we can already do a good deal of Boolean reasoning with $D_{T}^{+}$.

The Constant Rule $\{\neg\{c\} \mid c \in X\} \vdash_{\mathbb{X}}^{+} \perp_{\mathbb{X}}$ holds by (8.2) and part (4a) of Definition 8.1.

Lemma 8.6 For any edge $S \stackrel{\kappa}{\rightsquigarrow} S^{\prime}$ in $\operatorname{lng} T$, if $\Sigma$ is an $\mathcal{R}_{S^{-}}$-theory, then $[\kappa]^{-} \Sigma$ is an $\mathcal{R}_{S^{\prime}}$-theory.

Proof. First, $[\kappa]^{-} \Sigma$ is closed under Detachment, for if $\varphi, \varphi \rightarrow \psi \in[\kappa]^{-} \Sigma$, then $[\kappa] \varphi,[\kappa](\varphi \rightarrow$ $\psi) \in \Sigma$. But $[\kappa](\varphi \rightarrow \psi) \rightarrow([\kappa] \varphi \rightarrow[\kappa] \psi)$ is in $\Sigma$ by (8.1). But $\Sigma$ is closed under Detachment, so $\psi \in[\kappa]^{-} \Sigma$.

Next we show $A x_{S^{\prime}}^{+} \subseteq[\kappa]^{-} \Sigma$. If $\varphi \in A x_{S^{\prime}}^{+}$, then $[\kappa] \varphi \in A x_{S}^{+}$by Definition 8.1(5), so $[\kappa] \varphi \in \Sigma$ as $\Sigma$ is an $\mathcal{R}_{S^{-}}$theory. Hence $\varphi \in[\kappa]^{-} \Sigma$.

Finally we have to show that $[\kappa]^{-} \Sigma$ is $\mathcal{R}_{S^{\prime}}$-closed. Suppose $\langle\Gamma, \psi\rangle \in \mathcal{R}_{S^{\prime}}$ and $\Gamma \subseteq[\kappa]^{-} \Sigma$. Then $[\kappa] \Gamma \subseteq \Sigma$. But $\langle[\kappa] \Gamma,[\kappa] \psi\rangle \in \mathcal{R}_{S}$ by (4b) of Definition 8.1. Hence $[\kappa] \psi \in \Sigma$ and thus $\psi \in[\kappa]^{-} \Sigma$. 
Corollary 8.7 The Box Rule is satisfied by $D_{T}^{+}$.

Proof. $\quad$ Let $S \stackrel{\kappa}{\rightsquigarrow} S^{\prime}$ be an edge, and $\Gamma \vdash_{S^{\prime}}^{+} \psi$. To show $[\kappa] \Gamma \vdash_{S}^{+}[\kappa] \psi$, let $\Sigma$ be any $\mathcal{R}_{S^{-} \text {theory }}$ with $[\kappa] \Gamma \subseteq \Sigma$. Then $\Gamma \subseteq[\kappa]^{-} \Sigma$, and $[\kappa]^{-} \Sigma$ is an $\mathcal{R}_{S^{\prime}}$-theory by the Lemma, so $\psi \in[\kappa]^{-} \Sigma$ as $\Gamma \vdash_{S^{\prime}}^{+} \psi$, implying $[\kappa] \psi \in \Sigma$.

This completes the proof that $D_{T}^{+}$is a $T$-deduction system. To consider the Lindenbaum property we need

\section{Lemma 8.8}

(1) Every $\mathcal{R}_{S}$-theory is $\vdash_{S}^{+}$-closed.

(2) If a set $\Gamma: S$ of formulas is maximally finitely $\vdash_{S}^{+}$-consistent and $\mathcal{R}_{S}$-closed, then $\Gamma$ is $\vdash_{S}^{+}$-maximal.

Proof.

(1) If $\Gamma$ is an $\mathcal{R}_{S}$-theory and $\Gamma \vdash_{S}^{+} \varphi$, then as $\Gamma$ is an $\mathcal{R}_{S}$-theory extending $\Gamma$, the definition of $\vdash_{S}^{+}$immediately gives $\varphi \in \Gamma$.

(2) If $\Gamma$ is maximally finitely $\vdash_{S}^{+}$-consistent, then by (4) and (3) of Lemma 4.13 it is negation complete and closed under Detachment. Moreover, if $\varphi \in A x_{S}^{+}$then $\vdash_{S}^{+} \varphi$, so by Lemma 4.5(5) $\{\neg \varphi\}$ is not $\vdash_{S}^{+}$-consistent, hence $\{\neg \varphi\} \nsubseteq \Gamma$, and therefore $\varphi \in \Gamma$. Thus if $\Gamma$ is also $\mathcal{R}_{S}$-closed, then it is an $\mathcal{R}_{S}$-theory, hence is $\vdash_{S}^{+}$-closed by part (1). So if $\Gamma \vdash_{S}^{+} \perp_{S}$ then $\perp_{S} \in \Gamma$, contrary to the finite $\vdash_{S}^{+}$-consistency of $\Gamma$. Thus $\Gamma$ is $\vdash_{S}^{+}$-consistent and negation complete as required.

Theorem 8.9 For any Kripke polynomial functor $T$, if every rule-set $\mathcal{R}_{S}$ is countable, then $D_{T}^{+}$ is Lindenbaum and equal to Conseq $_{T}$.

Proof. Let $\left\langle\Sigma_{0}, \varphi_{0}\right\rangle,\left\langle\Sigma_{1}, \varphi_{1}\right\rangle, \ldots,\left\langle\Sigma_{n}, \varphi_{n}\right\rangle, \ldots$ be an enumeration of $\mathcal{R}_{S}$. We will use the fact that $\Sigma_{n} \vdash_{S}^{+} \varphi_{n}$ for all $n$, by (8.2).

Suppose $\Gamma: S$ is $\vdash_{S}^{+}$-consistent. Let $\Gamma_{0}=\Gamma$. Now assume inductively that $\Gamma_{n}: S$ is defined and $\vdash_{S}^{+}$-consistent. If $\Gamma_{n} \vdash_{S}^{+} \varphi_{n}$, then let

$$
\Gamma_{n+1}=\Gamma_{n} \cup\left\{\varphi_{n}\right\},
$$

which is $\vdash_{S}^{+}$-consistent because $\Gamma_{n}$ is, by Lemma 4.5(4). Alternatively, $\Gamma_{n} \nvdash_{S}^{+} \varphi_{n}$. But $\Sigma_{n} \vdash_{S}^{+} \varphi_{n}$, so in that case by the Cut Rule there must exist a $\psi \in \Sigma_{n}$ such that $\Gamma_{n} \nvdash_{S}^{+} \psi$. Let

$$
\Gamma_{n+1}=\Gamma_{n} \cup\{\neg \psi\},
$$

which is $\vdash_{S}^{+}$-consistent by Lemma $4.5(5)$.

Next, let $\Gamma_{\omega}=\bigcup_{n \geqslant 0} \Gamma_{n}$. By construction, for all $n$, if $\varphi_{n} \notin \Gamma_{\omega}$ then $\neg \psi \in \Gamma_{\omega}$ for some $\psi \in \Sigma_{n}$.

$\Gamma_{\omega}$ is finitely $\vdash_{S}^{+}$-consistent - any finite subset of $\Gamma_{\omega}$ is a subset of some $\Gamma_{n}$, which is $\vdash_{S}^{+}$ consistent. Since the union of any chain of finitely $\vdash{ }_{S}^{+}$-consistent sets is finitely $\vdash_{S}^{+}$-consistent, Zorn's Lemma then applies to the $\subseteq$-ordered set

$$
\left\{\Gamma^{\prime}: S \mid \Gamma_{\omega} \subseteq \Gamma^{\prime} \text { and } \Gamma^{\prime} \text { is finitely } \vdash_{S}^{+} \text {-consistent }\right\}
$$


to provide an extension $\Gamma^{\prime}$ of $\Gamma_{\omega}$ that is maximally finitely $\vdash_{S}^{+}$-consistent. (Alternatively, by a standard argument, we could enumerate Form ${ }_{S}$ and use Lemma 4.13(2) to proceed inductively along this (possibly transfinite) enumeration to build $\Gamma^{\prime}$ as a negation complete finitely $\vdash_{S}^{+}$ consistent extension of $\Gamma_{\omega}$.)

Now for each $n$, if $\varphi_{n} \notin \Gamma^{\prime}$ then $\varphi_{n} \notin \Gamma_{\omega}$ so by construction there exists $\psi \in \Sigma_{n}$ with $\neg \psi \in \Gamma_{\omega} \subseteq \Gamma^{\prime}$, hence $\psi \notin \Gamma^{\prime}$ or else $\{\neg \psi, \psi\}$ would contradict $\Gamma^{\prime}$ being finitely $\vdash_{S}^{+}$-consistent. This shows that $\Gamma^{\prime}$ is closed under the rule $\left\langle\Sigma_{n}, \varphi_{n}\right\rangle$ for all $n$, so is $\mathcal{R}_{S^{-}}$-closed. By Lemma 8.8(2) it follows that the extension $\Gamma^{\prime}$ of $\Gamma$ is $\vdash_{S}^{+}$-maximal.

This proves that for any $S \in \operatorname{lng}_{T}$, every $\vdash_{S}^{+}$-consistent set of $S$-formulas is included in some $\vdash_{S}^{+}$-maximal set, i.e. $D_{T}^{+}$is Lindenbaum. Since it is sound, its equality with the unique sound Lindenbaum system Conseq $_{T}$ follows.

The proof just given makes it plain as to why the countability of $\mathcal{R}_{S}$ is needed. As soon as $\Gamma_{\omega}$ is formed, we lose the $\vdash_{S}^{+}$-consistency of the $\Gamma_{n}$ 's, and retain only finite $\vdash_{S}^{+}$-consistency, so the construction could not be continued into the transfinite.

Corollary 8.10 If Form $_{S}$ is countable for all $S \in \operatorname{lng}_{T}$, then $D_{T}^{+}$is Lindenbaum and equal to Conseq $_{T}$.

Proof. It suffices to show that every rule-set $\mathcal{R}_{S}$ is countable.

The reader can confirm that an inference rule belongs to $\mathcal{R}_{S}$ iff it can be obtained from some constant rule of the type of Lemma 8.1(4a) by finitely many operations of the (4b) type $\langle\Gamma, \psi\rangle \mapsto\langle[\kappa] \Gamma,[\kappa] \psi\rangle$ and/or the (4c) type $\langle\Gamma, \psi\rangle \mapsto\langle\varphi \rightarrow \Gamma, \varphi \rightarrow \psi\rangle$. Note that although there are finitely many ingredients of $T$, these constructions could be repeatable ad infinitum if there are cycles in the multigraph of Ing $T$.

But if there are only countably many formulas altogether, then for any exponential ingredient $S^{E}$ there are only countably many formulas of type $\left[\mathrm{ev}_{e}\right] \varphi$, hence $E$ must be countable. Therefore there are countably many constructor symbols $\mathrm{pr}_{1}, \mathrm{pr}_{2}, \mathrm{in}_{1}, \mathrm{in}_{2}, \mathrm{ev}_{e}$, next, $\mathcal{P}$; so countably many box modalities $[\kappa]$. Also there are countably many formulas $\varphi$ that can be used to form a (4c) type rule $\langle\varphi \rightarrow \Gamma, \varphi \rightarrow \psi\rangle$. Thus there are countably many ways to form new rules, and finitely many constant rules to start from (one for each constant ingredient), so each $\mathcal{R}_{S}$ must be countable.

\section{Conclusion and Further Questions}

We have seen that a general theory of deduction systems can be developed for the logic of measurable and Kripke polynomial functors, and that this can be used to give a proof-theoretic construction of canonical coalgebras, leading to completeness theorems. The result is a characterisation of the logic determined by any functor as the least one satisfying the Lindenbaum property, and the only Lindenbaum one that is sound. In the case of KPF's we also saw that a particular system $D_{T}^{+}$can be defined that can be proven to be Lindenbaum when the language is countable.

Arising from this is the question of whether a version of $D_{T}^{+}$can be defined for measurable polynomial functors. This would require suitable closure conditions on $\mathcal{R}_{\Delta S}$, but even so there is an obstacle to proving the Countable Additivity Rule for $\vdash_{\Delta S}^{+}$by an argument similar to that for the Box Rule in Lemma 8.6 and Corollary 8.7. Closure of a set $\Sigma: \Delta S$ under Detachment does not imply closure of $[\geqslant p]^{-} \Sigma$ under Detachment, since the schema (8.1) is not valid when $\kappa$ is $\geqslant p$ with $0<p<1$, and so will not be included in $A x_{\Delta S}$. This matter requires further investigation.

A finitary KPF is a functor on Set that is built from the polynomial operations and the finitary powerset functor $\mathcal{P}_{\omega}$, where $\mathcal{P}_{\omega} X=\left\{Y \mid Y \subseteq_{\omega} X\right\}$. In [MV06] every finitary KPF 
is shown to have a final coalgebra whose "states" are the description sets of sort $I d$. A natural question here is whether there is a proof-theoretic version of this construction: can the description sets of coalgebras for a finitary KPF be characterised as the maximally consistent sets for some deduction system? Reflection on $\mathcal{P}_{\omega}$ itself indicates that this is problematic. A $\mathcal{P}_{\omega}$-coalgebra can be viewed as a Kripke frame $(X, R)$ that is image-finite, meaning that the point-image $R^{x}=\{y \in X \mid x R y\}$ of any point $x \in X$ is finite. The logic of $\mathcal{P}_{\omega}$-coalgebras can be handled by the variable-free one-sorted modal language of a single modality $\square$ (here Ing $\mathcal{P}_{\omega}$ looks like $I d \stackrel{\text { next }}{\rightsquigarrow} \mathcal{P}_{\omega} \stackrel{\mathcal{P}}{\rightsquigarrow} I d$, and $\square$ corresponds to [next] $\left.[\mathcal{P}]\right)$. The logic determined by the $\mathcal{P}_{\omega}$-coalgebras (i.e. the set of formulas valid in these coalgebras) is just the minimal normal $\square$-logic $\mathrm{K}$ in this language, since $\mathrm{K}$ is determined by the class of frames that are finite, hence image-finite. But the canonical frame $\mathcal{F}_{K}=\left(X_{\mathrm{K}}, R_{\mathrm{K}}\right)$ for $\mathrm{K}$ is not the final $\mathcal{P}_{\omega}$-coalgebra, since the description sets of image-finite frames form a proper subset $X_{\omega}$ of $X_{\mathrm{K}}$ that is $R_{\mathrm{K}}$-closed, i.e. $x \in X_{\omega}$ implies $R_{\mathrm{K}}^{x} \subseteq X_{\omega}$, and is itself image-finite. This can also be seen from an earlier characterisation of the final $\mathcal{P}_{\omega}$-algebra by Rutten [Rut95, p. 245]: it is given by the subset of $X_{\mathrm{K}}$ that is the union of the images of all bounded morphisms from image-finite frames to $\mathcal{F}_{K}$.

Now for each $n<\omega$, let Alt $_{n}$ be the set of all formulas of the form

$$
\square \varphi_{0} \vee \square\left(\varphi_{0} \rightarrow \varphi_{1}\right) \vee \cdots \vee \square\left(\varphi_{0} \wedge \cdots \wedge \varphi_{n-1} \rightarrow \varphi_{n}\right)
$$

[Seg71, page 52]. Let $\mathrm{K} n$ be the smallest normal logic including $\mathrm{Alt}_{n}$, and $X_{\mathrm{K} n}$ the set of all $\mathrm{K} n$-maximal sets of formulas. In any frame, $\mathrm{Alt}_{n}$ will be satisfied at any $x$ for which $R^{x}$ is of size at most $n$, and the canonical $\mathrm{K} n$ frame has the size of all its point-images bounded by $n$. The $X_{\mathrm{K} n}$ 's form an increasing sequence

$$
X_{\mathrm{K} 0} \subseteq X_{\mathrm{K} 1} \subseteq \cdots \subseteq X_{\mathrm{K} n} \subseteq \cdots \subseteq X_{\omega}
$$

But $\bigcup_{n} X_{\mathrm{K} n} \neq X_{\omega}$, as can be seen by considering descriptions sets of points in a suitable frame whose point-images are finite but unbounded in size.

Putting $X_{n}=\left\{x \in X_{\mathrm{K}} \mid\right.$ Alt $\left._{n} \subseteq x\right\}$, gives another increasing sequence $X_{0} \subseteq X_{1} \subseteq \cdots$ which covers $X_{\omega}$, i.e. $X_{\omega} \subseteq \bigcup_{n} X_{n}$. For each $n$ we have $X_{\omega} \nsubseteq X_{n}$, as can be seen from image-finite frames with point-images larger than $n$. Moreover $X_{1} \nsubseteq X_{\omega}$, as can be seen from a suitable frame with an $x$ having $R^{x}=\{y\}$ and $R^{y}$ infinite. Hence $X_{\omega} \neq \bigcup_{n} X_{n}$.

These observations cast some doubt on whether there is a natural set of syntactic closure conditions that characterises the image-finiteness of the members of $X_{\omega}$.

A similar issue arises with the discrete probability measure functor $\mathcal{D}:$ Set $\rightarrow$ Set, where $\mathcal{D} X$ is the set of all functions $\mu: X \rightarrow[0,1]$ whose support $\{x \in X \mid \mu(x)>0\}$ is finite, and $\sum_{x \in X} \mu(x)=1$. [MV06] shows that there is a final coalgebra for every functor built from the polynomial operations, $\mathcal{P}_{\omega}$ and $\mathcal{D}$. It is not clear what syntactic closure conditions might capture the finiteness of support of a discrete probability measure.

There has been recent interest [ED06] in modal logic for systems associated with the subcategory of Meas comprising the analytic spaces, those that are continuous images of Polish spaces. The functor $\Delta$ is replaced by the subprobability functor $\mathfrak{S}$, where $\mathfrak{S} \mathbb{X}$ is the space of measures on $\mathbb{X}$ having $\mu(X) \leqslant 1$. It would be of interest to investigate deduction systems and canonical models in this setting.

Finally, since $\sigma$-algebras are closed under countable unions and intersections, it would be possible to work with modal languages that are closed under countable disjunctions and conjunctions. Completeness theorems for such infinitary languages typically work with countable fragments of the set of formulas. This may provide a setting for a coalgebraic application of the Loeb measure construction from nonstandard analysis, as in [Hoo78]. 


\section{References}

[Acz77] Peter Aczel. An introduction to inductive definitions. In Jon Barwise, editor, Handbook of Mathematical Logic, pages 739-782. North-Holland, 1977.

[Aum99] Robert J. Aumann. Interactive epistemology II: Probability. International Journal of Game Theory, 28:301-314, 1999.

[ED06] Ernst Erich Doberkat. Stochastic relations: Congruences, bisimulations and the Hennessy-Milner theorem. SIAM Journal on Computing, 35(3):590-626, 2006.

[FG06] David Friggens and Robert Goldblatt. A modal proof theory for final polynomial coalgebras. Theoretical Computer Science, 360:1-22, 2006.

[Gol82] Robert Goldblatt. Axiomatising the Logic of Computer Programming, volume 130 of Lecture Notes in Computer Science. Springer-Verlag, 1982.

[Gol93] Robert Goldblatt. A framework for infinitary modal logic. In Mathematics of Modality, CSLI Lecture Notes No. 43, pages 213-229. CSLI Publications, Stanford, California, 1993.

[Hal74] Paul R. Halmos. Measure Theory, volume 18 of Graduate Texts in Mathematics. Springer, 1974.

[HM01] Aviad Heifetz and Philippe Mongin. Probability logic for type spaces. Games and Economic Behavior, 35:31-53, 2001.

[Hoo78] Douglas N. Hoover. Probability logic. Annals of Mathematical Logic, 14:287-313, 1978.

[HS98] Aviad Heifetz and Dov Samet. Topology-free typology of beliefs. Journal of Economic Theory, 82:324-341, 1998.

[Jac01] Bart Jacobs. Many-sorted coalgebraic modal logic: a model-theoretic study. Theoretical Informatics and Applications, 35:31-59, 2001.

[MV04] Lawrence S. Moss and Ignacio D. Viglizzo. Harsanyi type spaces and final coalgebras constructed from satisfied theories. Electronic Notes in Theoretical Computer Science, 106:279-295, 2004.

[MV06] Lawrence S. Moss and Ignacio D. Viglizzo. Final coalgebras for functors on measurable spaces. Information and Computation, 204:610-636, 2006.

[Röß00] Martin Rößiger. Coalgebras and modal logic. Electronic Notes in Theoretical Computer Science, 33, 2000.

[Rut95] J. J. M. M. Rutten. A calculus of transition systems (towards universal coalgebra). In Alban Ponse, Maarten de Rijke, and Yde Venema, editors, Modal Logic and Process Algebra, CSLI Lecture Notes No. 53, pages 231-256. CSLI Publications, Stanford, California, 1995.

[Rut00] J.J.M.M. Rutten. Universal coalgebra: a theory of systems. Theoretical Computer Science, 249(1):3-80, 2000.

[Seg71] Krister Segerberg. An Essay in Classical Modal Logic, volume 13 of Filosofiska Studier. Uppsala Universitet, 1971. 
[Sri98] S. M. Srivastava. A Course on Borel Sets, volume 180 of Graduate Texts in Mathematics. Springer, 1998.

[Vig05] Ignacio D. Viglizzo. Coalgebras on Measurable Spaces. PhD thesis, Indiana University, 2005. 